\title{
Evaluating whole-genome sequencing quality metrics for enteric pathogen outbreaks
}

\author{
Darlene D Wagner $^{1,2}$, Heather A Carleton ${ }^{3}$, Eija Trees ${ }^{4}$, Lee S Katz ${ }^{\text {Corresp. } 3,5}$ \\ ${ }^{1}$ Division of Viral Diseases, Centers for Disease Control and Prevention, Atlanta, GA, United States of America \\ 2 Eagle Medical Services, LLC, Atlanta, GA, United States of America \\ 3 Enteric Diseases Laboratory Branch, Centers for Disease Control and Prevention, Atlanta, GA, United States of America \\ 4 Association of Public Health Laboratories, Silver Spring, MD, United States of America \\ ${ }^{5}$ Center for Food Safety, University of Georgia, Griffin, GA, United States of America \\ Corresponding Author: Lee S Katz \\ Email address: gzu2@cdc.gov
}

Background. Whole genome sequencing (WGS) has gained increasing importance in responses to enteric bacterial outbreaks. Common analysis procedures for WGS, single nucleotide polymorphisms (SNPs) and genome assembly, are highly dependent upon WGS data quality.

Methods. Raw, unprocessed WGS reads from Escherichia coli, Salmonella enterica, and Shigella sonnei outbreak clusters were characterized for four quality metrics: PHRED score, read length, library insert size, and ambiguous nucleotide composition. PHRED scores were strongly correlated with improved SNPs analysis results in $E$. coli and $S$. enterica clusters.

Results. Assembly quality showed only moderate correlations with PHRED scores and library insert size, and then only for Salmonella. To improve SNP analyses and assemblies, we compared seven read-healing pipelines to improve these four quality metrics and to see how well they improved SNP analysis and genome assembly. The most effective read healing pipelines for SNPs analysis incorporated quality-based trimming, fixed-width trimming, or both. The Lyve-SET SNPs pipeline showed a more marked improvement than the CFSAN SNP Pipeline, but the latter performed better on raw, unhealed reads. For genome assembly, SPAdes enabled significant improvements in healed $E$. coli reads only, while Skesa yielded no significant improvements on healed reads.

Conclusions. PHRED scores will continue to be a crucial quality metric albeit not of equal impact across all types of analyses for all enteric bacteria. While trimming-based read healing performed well for SNPS analyses, different read healing approaches are likely needed for genome assembly or other, emerging WGS analysis methodologies. 


\section{Evaluating Whole-Genome Sequencing Quality 2 Metrics for Enteric Pathogen Outbreaks}

3

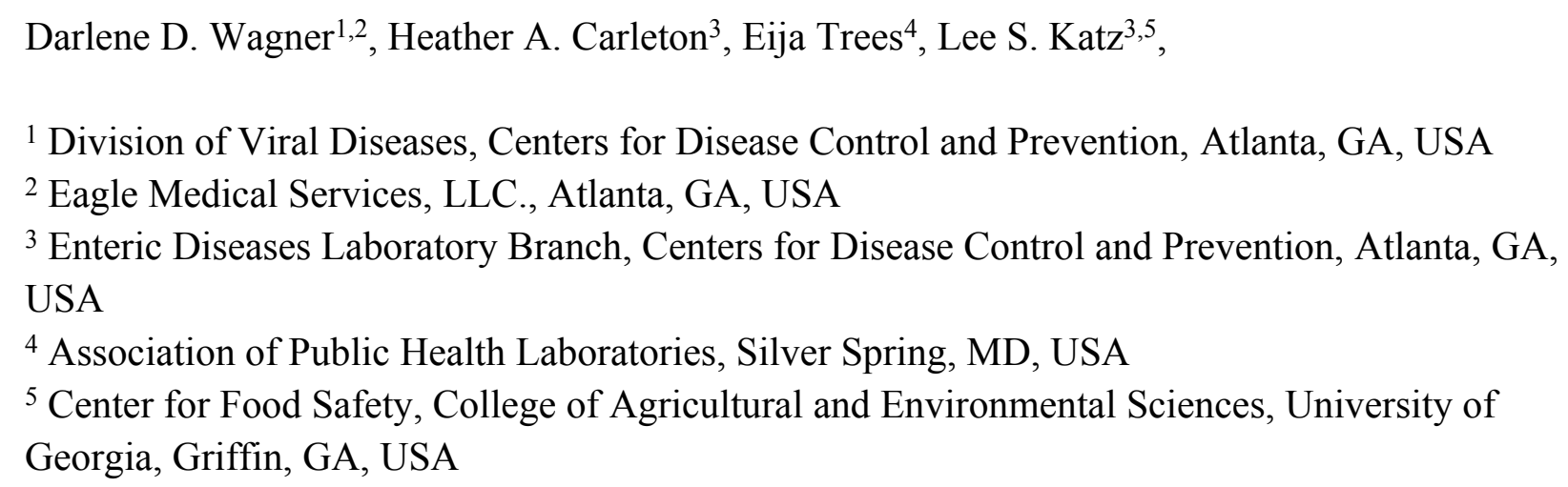




\section{Abstract}

Whole genome sequencing (WGS) has gained increasing importance in responses to enteric bacterial outbreaks. Common analysis procedures for WGS, single nucleotide polymorphisms (SNPs) and genome assembly, are highly dependent upon WGS data quality. Raw, unprocessed WGS reads from Escherichia coli, Salmonella enterica, and Shigella sonnei outbreak clusters were characterized for four quality metrics: PHRED score, read length, library insert size, and ambiguous nucleotide composition. PHRED scores were strongly correlated with improved SNPs analysis results in E. coli and $S$. enterica clusters. Assembly quality showed only moderate correlations with PHRED scores and library insert size, and then only for Salmonella. To improve SNP analyses and assemblies, we compared seven read-healing pipelines to improve these four quality metrics and to see how well they improved SNP analysis and genome assembly. The most effective read healing pipelines for SNPs analysis incorporated qualitybased trimming, fixed-width trimming, or both. The Lyve-SET SNPs pipeline showed a more marked improvement than the CFSAN SNP Pipeline, but the latter performed better on raw, unhealed reads. For genome assembly, SPAdes enabled significant improvements in healed $E$. coli reads only, while Skesa yielded no significant improvements on healed reads. PHRED scores will continue to be a crucial quality metric albeit not of equal impact across all types of analyses for all enteric bacteria. While trimming-based read healing performed well for SNPs analyses, different read healing approaches are likely needed for genome assembly or other, emerging WGS analysis methodologies.

\section{Introduction}

Whole genome sequencing (WGS) has become instrumental to studies of enteric bacteria ranging from outbreak detection to microbial ecology. WGS data are analyzed by single nucleotide polymorphisms (SNPs), genome assembly, and multi-locus sequence typing (MLST). SNPs enable high-resolution quantification of nucleotide variation among microbial isolates, often by mapping the reads of WGS data to a reference genome. SNP analyses have proven indispensable to solving several high profile foodborne, waterborne, and domesticated animal outbreaks (Crowe et al. 2017; Katz et al. 2013; Marshall et al. 2018).

Genome assembly brings overlapping WGS reads together into contiguous blocks representing the original genome sequence (i.e. contigs). Genome assembly serves as a first step for many pipelines which characterize genes involved in antibiotic resistance and virulence (Singh et al. 2018; Wang et al. 2017) as well as elucidating phylogenetic relatedness through average nucleotide identity (Cheleuitte-Nieves et al. 2018; Goris et al. 2007). Given that SNPs and genome assembly are widespread analysis procedures, they can strongly impact the reliability of WGS data interpretation for clinical microbiology and epidemiologic investigations. In turn, the quality of SNPs and assemblies depend upon the quality of the original WGS reads. 
73 WGS quality control is thus an ongoing challenge for the multiple stakeholders involved in

74 outbreak responses and evolutionary biology of enteric bacteria (Moran-Gilad et al. 2015;

75 Timme et al. 2018). Characterization of WGS quality artifacts and quality metrics will build

76 additional confidence in WGS methods in clinical microbiology and molecular epidemiology.

77 WGS quality metrics encompass read length, library insert size, PHRED scores, and base

78 pair composition. The PHRED score system assigns a quality, $\mathrm{q}(\mathrm{q}=-10 \cdot \log 10(\mathrm{p}))$, to each base

79 in a WGS data file, denoting the probability of a miscall (Ewing \& Green 1998). At present,

80 Illumina platforms dominate the market of short-read WGS technologies. Low PHRED scores

81 are the most common quality issue encountered in Illumina data. Average quality scores

82 typically decrease towards the 3' ends of reads (Dohm et al. 2008; Minoche et al. 2011). In

83 addition, Illumina reverse reads exhibit lower overall quality relative to forward reads (Guo et al.

84 2012; Tan et al. 2019). Given the correlation between PHRED scores and base miscalls, low

85 PHRED scores are associated with more fragmented genome assemblies (Smeds \& Künstner

86 2011) and less reliable SNP discovery (Guo et al. 2012). Library insert size (also referred to as

87 "insert length" in this manuscript) is another WGS-quality metric shown to be critical to bacterial

88 genomics (Chen et al. 2015; Huptas 2016). When insert lengths are shorter than read lengths,

89 adapter sequences may occur in the WGS data set and average read length will be reduced

90 (Turner 2014). For bacterial genome assembly, increased insert lengths provide less fragmented,

91 higher quality assemblies (Chen et al. 2015; Clooney et al. 2016; Head et al. 2014; Huptas 2016;

92 Trivedi et al. 2014). By contrast, insert lengths have shown less predictable impacts on SNPs

93 analyses. One study employed short inserts with overlapping reads to enhance SNP calling

94 accuracy (Chen-Harris et al. 2013). On the other hand, large insert sizes help identify genomic

95 insertions, deletions, and rearrangements (Guan \& Sung 2016).

96 Read healing comprises any processing method applied to WGS reads to improve overall

97 quality prior to their use in SNPs, genome assembly, or any other computational analysis. To

98 mitigate the effects of low PHRED quality and other artifacts in raw WGS reads, dozens of

99 open-source software tools have become available to perform read healing (Fabbro et al. 2013;

100 Heydari et al. 2017; Patel \& Jain 2012). There are three broad categories of read-healing tools,

101 filtering, trimming, and error correction (Smeds \& Künstner 2011). The filtering strategy

102 removes entire reads failing a given quality threshold. The Illumina chastity filter is an example

103 of the filtering approach (Whiteford et al. 2009). By comparison, trimming removes portions of

104 reads which fail quality thresholds or distance thresholds from the 5'/3' ends of the reads.

105 Prinseq and Fastx-toolkit are examples of tools implementing filtering, trimming, or both

106 (Hannon 2010; Patel \& Jain 2012; Schmieder \& Edwards 2011). The error correction strategy

107 employs kmer or alignment techniques to correct miscalls in reads. Quake and BayesHammer

108 provide examples of read healing by correction (Kelley et al. 2010; Nikolenko et al. 2013).

109 While many previous studies have compared read healing tools, few have characterized

110 how quality metrics and read healing correlate within bacterial WGS sets representing outbreaks.

111 The current study describes effects of quality metrics and read healing upon the Illumina WGS

112 data representing four enteric bacterial outbreaks. This study also describes correlations between 
113 WGS quality, SNPs analysis, and genome assemblies for the outbreak data sets (Table S1).

114 Admittedly, the results of this study are limited to neutral GC content organisms and do not

115 include high or low GC content organisms such as Listeria. Filtering and trimming approaches

116 to read healing elucidate the WGS quality metrics imparting the greatest effect upon intermediate

117 analyses. Finally, the most effective healing techniques are recommended for addressing quality

118 artifacts and improved intermediate analyses.

119

120

121

122

123

124

125

126

127

128

129

130

131

132

133

134

135

136

137

138

139

140

141

142

143

144

145

146

147

148

149

150

\section{Materials \& Methods}

Isolates from four outbreak-associated Enterobacterales clusters were evaluated. All sequencing was performed on the Illumina MiSeq (San Diego, CA) using the Nextera XT (Illumina) library preparation. Isolates were collected over the span of several months to represent outbreak clusters and were submitted by multiple public health laboratories. As a consequence, 300-, 500- and/or 600-cycle sequencing chemistries were represented in each data cluster. The four outbreak clusters represented Escherichia coli O26 (Cluster 1, Table S1 A), Salmonella enterica serovar Reading (Cluster 2, Table S1 B), S. enterica serovar Pomona (Cluster 3, Table S1 C, (Gambino-Shirley et al. 2018; Walters et al. 2016)), and Shigella sonnei (Cluster 4, Table S1 D). These predicted outbreak clusters were chosen due to the presence of quality artifacts such has high proportions of ambiguous nucleotides. The use of GC-neutral Enterobacterales genomes were expected to minimize GC-composition artifacts (Laehnemann et al. 2016) for single nucleotide polymorphisms (SNPs) and assemblies analysis.

Sequencing reads were analyzed for quality. Raw reads were checked for contamination using the standard Kraken1 database in Kraken v1.0.0; no conflicting taxonomies were detected (Wood \& Salzberg 2014). Raw reads were down-sampled to yield near uniform genome coverage within each of the four clusters prior to PHRED score analysis using CG-Pipeline (Ewing \& Green 1998; Kislyuk et al. 2010)(Table S2). Reads with ambiguous nucleotides, Ns, in down-sampled isolates were counted using a custom python wrapper for prinseq-lite.pl (Schmieder \& Edwards 2011)(Table S2). Full-coverage raw reads were processed through each of seven read-healing pipelines, followed by down-sampling, and estimation of quality metrics. To control for sequencing coverage of isolates, all raw and healed E. coli O26 (Cluster 1) reads were down-sampled to 50X coverage, $S$. enterica serovar Reading (Cluster 2) reads were downsampled to 65X, S. enterica serovar Pomona (Cluster 3) reads were down-sampled to 58X, and S. sonnei (Cluster 4) reads were down-sampled to 60X using CG-pipeline scripts (Table S2). Sets could not be normalized to higher coverages without excluding isolates from clusters.

Sequence reads from isolates in all four clusters were each healed through seven pipelines or else were left raw for comparison. Four of the pipelines were based upon a single computational step, either read length filtering, quality trimming, blunt-end trimming, or error correction (Table S3). The prinseq-lite wrapper, windowQualPrinseqLite_R1andR2.py (github.com/darlenewagner/NGS_Multi_Heal (version 0.9)), was used to implement the pipeline 
151

152

153

154

155

156

157

158

159

160

161

162

163

164

165

166

167

168

169

170

171

172

173

174

175

176

177

178

179

180

181

182

183

184

185

186

187

188

189

190

'noNmin 100', which filtered out reads based upon length and presence of ambiguous nucleotides (Table S3). The fastx_trimmer wrapper script, fastxQualAdaptTrimmer_R1andR2.py, implemented the pipeline, 'fastxOnly-3pr', and trimmed a fixed number of bases from the 3' ends of reads using FASTX-Toolkit (Hannon 2010) (Table S3). The prinseq-lite wrapper, windowQualPrinseqLite_R1andR2.pl, also implemented trimming based upon PHRED quality and read length/ambiguous nucleotide filtering (prinseq in Table S3). The remaining single step read-healing pipeline, 'BayesHammer' implemented SPAdes v. 3.6.1 at the setting '--only-errorcorrection' to perform a Bayesian sub-clustering error correction (Nikolenko et al. 2013). Briefly, BayesHammer's error correction is based upon the number of changes required to match aligned subsequences. The three remaining pipelines were comprised of two-steps. The pipeline, 'noNmin 100-3pr', combined the filtering parameters of noNmin 100 with the 3' bluntend trimming of fastxOnly-3pr (Table S3). The pipeline, 'prinseq-3pr', combined the parameters of 'prinseq' with a 3' blunt-end trim. A related pipeline, 'prinseq-5pr3pr', had the same parameters as prinseq-3pr but with an additional 5' trim from both R1 and R2 reads (Table S3). All of our trimming pipelines consist of removal of $3^{\prime}$ and/or 5' ends of reads, which might be presumably lower-quality. In summary, six of the healing pipelines healed reads based upon three quality metrics: PHRED quality, ambiguous nucleotide composition, and read length. The seventh pipeline, BayesHammer, healed reads through correction of base miscalls (Nikolenko et al. 2013).

For each combination of outbreak clusters and read healing pipelines, read healing effects on high-quality single nucleotide polymorphisms (SNPs) analysis were evaluated. Multiple sequence alignments based upon genomic SNPs were constructed using the Lyve-SET v1.1.4f (Katz et al. 2017) and the CFSAN SNP Pipeline version 1.0.1 (Davis et al. 2015). For the E. coli O26 (Cluster 1) isolates, the closed chromosome of strain 11368 (accession AP010953) was used as the Lyve-SET and CFSAN SNP Pipeline reference. For Salmonella Reading (Cluster 2), no closely related finished reference was available, so an assembly from one of the study isolates, CVM_N17S1020, was used as an internal reference. For the Pomona cluster, the PacBio chromosome, 2012K-0678 (accession CP020718), served as reference. For the Shigella cluster, the PacBio chromosome, 2015C-3794 (accession CP022455), served as reference. Prophage regions were detected using PHASTER (Arndt et al. 2016) and regions flagged as phage-derived were masked using the custom script, mask_prophages_within_chromosome.pl

(https://github.com/darlenewagner/NGS_Multi_Heal, (version 0.9)). Masked prophage regions used in the three closed reference chromosomes in the study are shown in Table S4. For all four clusters, Lyve-SET filtering parameters were set to remove SNPs with less than 20X coverage, less than $95 \%$ consensus, and occurring within less than 5 bp of neighboring SNPs. In the CFSAN SNP Pipeline, the default filtering parameters were employed, $8 \mathrm{X}$ coverage and $90 \%$ consensus (Davis et al. 2015). Proportions of reads mapped to references were obtained using 'flagstat' in samtools-0.1.19 (Li et al. 2009). True positive SNPs count for method $\mathrm{i}, \mathrm{TP}_{\mathrm{i}}$, were estimated from the intersection of SNPs positions healed by three other methods, $S_{j}, S_{k}$, and $S_{l}$, and the intersection of method $\mathrm{i}, \mathrm{S}_{\mathrm{i}}$. 
191

192

193

194

195

196

197

198

199

200

201

202

203

204

205

206

207

208

209

210

211

212

213

214

215

216

217

218

219

220

221

222

223

224

225

226

227

228

$\mathrm{TP}_{\mathrm{i}}=\left|\mathrm{S}_{\mathrm{i}} \cap\left(\mathrm{S}_{\mathrm{j}} \cap \mathrm{S}_{\mathrm{k}} \cap \mathrm{S}_{\mathrm{l}}\right)\right|$

False discoveries for method $\mathrm{j}, \mathrm{FP}_{\mathrm{j}}$, were estimated by taking the set difference between SNP positions of method $j, S_{j}$, and the union of SNP positions in all other methods.

$F P_{j}=\left|S_{j}-\left(S_{1} \cup \ldots \cup S_{i \neq j} \cup \ldots \cup S_{7}\right)\right|$

Draft assemblies were built for each sequenced isolate using raw reads and the seven sets of healed reads. Initial assemblies were performed in SPAdes v3.6.1 (Bankevich et al. 2012) using four bash wrapper scripts each with 'careful' assembly and differing $k$-mer combinations. The wrapper scripts employed seven (-k 21, 35, 45, 55, 75, 95, 121), eight (-k 21, 33, 43, 51, 63, $73,99,123)$ or nine $(-\mathrm{k} 21,33,45,55,63,77,99,105,121$ and $21,35,49,57,63,77,99,105$, 121) $k$-mer bin combinations. In this manner, raw and healed reads of each isolate was assembled four times; the assembly with the lowest number of contigs with lengths $>500 \mathrm{bp}$ was selected using the custom script, multiFastaContigAvgJudgement.py

(https://github.com/darlenewagner/NGS_Multi_Heal (version 0.9)). Assemblies of equal contig count were selected based upon higher average contig length; assemblies of equal average contig length were selected based upon higher N50. Raw or healed reads were mapped using SMALT v 0.7.6 ((Ponstingl \& Ning 2010), https://www.sanger.ac.uk/science/tools/smalt-0) with settings as employed previously (Katz et al. 2017). Insert sizes were inferred from SMALT mappings of raw or healed reads onto their respective best-of-four draft SPAdes assemblies. Read lengths and pair distances were extracted from resulting SAM files using samtools (Li et al. 2009). An additional script, cLikeFastaStats.py (https://github.com/darlenewagner/NGS_Multi_Heal (version 0.9)) was used to calculate assembly quality metrics such as contig count, N50, and maximum contig length. To compare the best SPAdes assembly against an alternate assembler, each isolate was assembled once each in Skesa using default parameters (Souvorov et al. 2018). Improvements in read metrics, mapping metrics, and assembly metrics were evaluated using the Kruskal-Wallis rank sum test and Dunn post-hoc tests in the R packages, dplyr and FSA, respectively. To adjust p-values for multiple comparisons, the Benjamani-Hochberg correction was used through the FSA R package. Spearman correlation $\left(\mathrm{r}_{\mathrm{s}}\right)$ was computed using the $\mathrm{R}$ base package. All quality metrics were evaluated in comparison to raw sequence reads for the individual isolate.

\section{Results}

\section{Quality metrics in raw and healed reads.}

Paired-end Illumina reads from isolates within Cluster 1 (E. coli) varied widely with respect to quality metrics (Table 1, Data S1). Eighteen of the isolates, sequenced using 500cycle chemistry (Figure 1), had read lengths ranging from 35 to $250 \mathrm{bp}$ (average $=212.4 \mathrm{bp}$ ). Median insert sizes for 500-cycle reads ranged from $182 \mathrm{bp}$ up to $371 \mathrm{bp}$ (average of medians = $273.4 \mathrm{bp}$ ). Average read lengths tended to increase with increasing median insert lengths among isolates sequenced on 500-cycle chemistry with a robust Spearman correlation $\left(r_{s}=0.792\right)$.

Peer) reviewing PDF | (2021:05:60850:1:1:NEW 10 Sep 2021) 
229 Forward (R1) reads sequenced by 500-cycle chemistry had average PHRED qualities of $34.9(\sigma$ $230=0.73)$. Reverse reads (R2) exhibited lower PHRED scores, averaging $30.6(\sigma=2.1)$, with one 231 isolate as low as 26.0 (Table 1, top left). PNUSAE001785 had at least one Ns position on $10.3 \%$ 232 of R2 reads while 2014C-3572 had Ns positions in 3.4\% of both R1 and R2 reads (Supplemental 233 Data S1). R1 and R2 PHRED scores both exhibited a weak correlation with ambiguous 234 nucleotide composition $\left(\mathrm{r}_{\mathrm{s}}=-0.0596\right.$ and -0.180$)$. The remaining two isolates in the $\mathrm{O} 26$ cluster, 235 PNUSAE001782 and PNUSAE001783, were sequenced using 600-cycle chemistry (Figure 1). 236 The 600 -cycle read lengths ranged from $35 \mathrm{bp}$ to $300 \mathrm{bp}$ (average $=224.5 \mathrm{bp}$ ) with R2 PHRED 237 quality averages around 27.7. The 600-cycle isolates showed the lowest median insert lengths in 238 the cluster, $191 \mathrm{bp}$ for PNUSAE001782 and $161 \mathrm{bp}$ for PNUSAE001783 (Table 1).

239

240

241

242

243

244

245

246

247

248

249

250

251

252

253

254

255

256

257

258

259

260

261

262

263

264

265

266

267

268
After healing, R1 and R2 PHRED scores, along with ambiguous nucleotide count, showed significant improvement. Together, prinseq, prinseq-3pr, and prinseq-5pr3pr significantly improved R1 scores and R2 scores, indicated by Dunn test post-hoc $p$-values under 0.05 (Table S5, Data S1, $\mathrm{df}=7$ ). Overall, the prinseq, prinseq-3pr, and prinseq-5pr3pr pipelines increased average R2 quality by up to three points (Table 1). Ambiguous nucleotide content significantly decreased under prinseq, prinseq-5pr3pr, and prinseq-3pr (Table S5). The Dunn test indicated average read lengths significantly decreasing under prinseq, prinseq-3pr, and prinseq-3pr5pr $\left(2.81 \times 10^{-5}<p<6.43 \times 10^{-4}\right)$. For example, prinseq-5pr3pr decreased average read lengths by 25 bp relative to raw reads. No healing method significantly changed insert lengths. In summary, prinseq, prinseq-3pr, and prinseq-5pr3pr combined improved PHRED scores with a decrease in read lengths.

Paired-end reads from isolates of Cluster 2 (S. enterica serovar Reading) exhibited quality metrics values comparable to Cluster 1. R1 PHRED quality averaged around 34.2 while R2 PHRED quality averaged roughly 4 points lower (Table 1, Data S2). Out of twenty-one isolates in the Reading cluster, twelve had R2 PHRED quality scores below 30.0 (mean of averages $=30.2, \sigma=1.5$, Table 1). Median insert sizes per isolate varied from $175 \mathrm{bp}$ to $531 \mathrm{bp}$ (average $=279, \sigma=86.1$, Table 1). Thirteen isolates in the cluster had median insert lengths below $300 \mathrm{bp}$, including the four isolates sequenced by 600-cycle chemistry (218 bp to $253 \mathrm{bp}$ ). Average read lengths for 500-cycle chemistry ranged from $181 \mathrm{bp}$ to $245 \mathrm{bp}$, while 600 -cycle chemistry read lengths ranged from $228 \mathrm{bp}$ to $245 \mathrm{bp}$. Isolates with longer read lengths also tended to have longer insert lengths, indicated by a moderate correlation $\left(r_{s}=0.604\right)$. Counting only the 500-cycle chemistry reads, this correlation increased to $r_{s}=0.953$. Six of the Reading cluster isolates, including three of the isolates sequenced with 600-cycle chemistry, had Ns present on more than $1.0 \%$ of R1 and/or R2 reads (Supplemental Data S2). R2 PHRED exhibited a slight positive correlation with percentage of Ns in $R 2$ reads $\left(r_{s}=0.301\right)$, while R1 PHRED had a negligible association with R1 Ns $\left(\mathrm{r}_{\mathrm{s}}=0.0565\right)$.

Read healing in the Cluster 2 yielded improvement in R1/R2 PHRED and percent ambiguous nucleotides. Prinseq-3pr and prinseq-5pr3pr yielded the largest R2 PHRED score increases (mean of averages $=33.4$ and 33.6, Table 1); for 12 out of the 21 isolates, these pipelines raised R2 scores by at least 4 points. Only prinseq, prinseq-3pr, and prinseq-5pr3pr

Peer) reviewing PDF | (2021:05:60850:1:1:NEW 10 Sep 2021) 
269

270

271

272

273

274

275

276

277

278

279

280

281

282

283

284

285

286

287

288

289

290

291

292

293

294

295

296

297

298

299

300

301

302

303

304

305

306

307

308

significantly raised both R1 and R2 PHRED scores under the Dunn post hoc test (Table S5, df= 7). Insert lengths showed no improvement under the Kruskal-Wallis test. The Dunn post hoc test indicated that three out of the seven pipelines, prinseq, prinseq- $3 \mathrm{pr}$, and prinseq- $5 \mathrm{pr} 3 \mathrm{r}$, significantly reduced read length $\left(2.570 \times 10^{-6}<p<2.601 \times 10^{-5} ; \mathrm{df}=7\right)$. For instance, prinseq$5 \mathrm{pr} 3 \mathrm{pr}$ reduced read length by an average of $33 \mathrm{bp}$ below the $221 \mathrm{bp}$ average for raw reads. In this manner, healing pipelines which increased R1 and R2 PHRED scores also reduced read lengths.

Paired-end reads for isolates of Cluster 3 (S. enterica serovar Pomona) exhibited the widest variation in quality metrics among all clusters analyzed. Average R2 PHRED quality across the 25 Pomona isolates ranged from 26.1 to 35.3 (mean $=32.2, \sigma=3.1$, Table 1 , Data S3). R1 PHRED quality averaged 4 points higher (mean $=35.3, \sigma=1.6$, Table 1$)$ than R2 scores. Median insert sizes varied widely from $182 \mathrm{bp}$ to $556 \mathrm{bp}($ mean $=383.4, \sigma=107.4$, Table 1$)$. Average read length and median insert length showed a moderate correlation among the twenty isolates sequenced with 500-cycle chemistry $\left(r_{\mathrm{s}}=0.693\right)$. The two isolates sequenced with 600cycle chemistry, PNUSAS002454 and PNUSAS002455, showed robust read and insert lengths

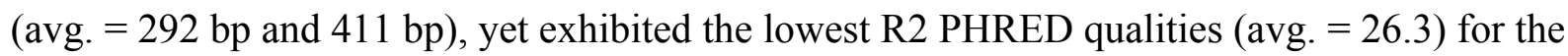
cluster. The three isolates sequenced with 300-cycle chemistry (Figure 1) had the highest R2 PHRED scores, ranging from at 35.3 to 36.6 (Table 1). At the same time, the 300 -cycle sequenced isolate, 2015EL-1657B, had ambiguous nucleotides (Ns) on $64 \%$ of its R2 reads (Table 1, Supplemental Data S3). Ns counts were high on two of the 500-cycle sequences: 2016K-0057 had 3\% of R1 reads with Ns and PNUSAS002458 had 10\% of R2 reads with Ns. R1 and R2 PHRED scores exhibited weak correlations with Ns content ( $r_{s}=-0.304$ and -0.114$)$.

R2 PHRED quality and ambiguous nucleotide percentage were the only quality metrics in the Pomona cluster to show significant improvement. Only prinseq, prinseq-3pr, and prinseq5pr3pr significantly improved R2 PHRED under Dunn's post hoc test $(0.0151<\mathrm{p}<0.0230$, Table S5). Prinseq-5pr3pr and prinseq-3pr both increased average R2 PHRED scores across the cluster by over 2 points, to 34.4 (Table 1). Ambiguous nucleotide percentages also significantly decreased after prinseq, prinseq-5pr3pr, and prinseq-3pr healing $\left(1.66 \times 10^{-6}<p<2.30 \times 10^{-6}\right)$. Prinseq-3pr reduced PNUSAS002458 reads N-counts from 10.0\% to $1.4 \%$ and reduced 2015EL1657B (300-cycle) N-counts from 63\% to less than 1\%. Neither R1 PHRED nor median insert lengths exhibited significant improvements under the Kruskal-Wallis test ( $\mathrm{df}=7$, Table S5). Prinseq-3pr, and prinseq-5pr3pr reduced average reads lengths to 197 and $198 \mathrm{bp}$, down from the $224 \mathrm{bp}$ average for raw reads. This change was significant under the Dunn's post hoc test (3.49 $\left.\times 10^{-4}<p<4.81 \times 10^{-4}\right)$. In this manner, improvements in read quality were accompanied by decreases in read lengths.

Paired-end reads of Cluster 4 ( $S$. sonnei) exhibited robust R1 and R2 PHRED quality metrics but wide variability in read lengths, insert lengths, and ambiguous nucleotide content. Average R2 PHRED quality across the 21 Cluster 4 isolates ranged from 30.2 to 35.4 (mean = 33.9, $\sigma=1.4$, Table 1, Data S4), while R1 PHRED scores averaged higher ( mean $=35.9, \sigma=0.3$, Table 1). Median insert sizes ranged from $212 \mathrm{bp}$ to $492 \mathrm{bp}($ mean $=309.3 \mathrm{bp} ; \sigma=86.6 \mathrm{bp}$, 
309

310

311

312

313

314

315

316

317

318

319

320

321

322

323

324

325

326

327

328

329

330

331

332

333

334

335

336

337

338

339

340

341

342

343

344

345

346

347

Table 1) with 11 isolates below 300bp. All Cluster 4 isolates were sequenced by 500 -cycle chemistry (Figure 1) and had average lengths ranging from $191.5 \mathrm{bp}$ to $245.7 \mathrm{bp}$. Average read lengths and median insert lengths were strongly correlated $\left(\mathrm{r}_{\mathrm{s}}=0.964\right)$. Four isolates had ambiguous nucleotides (Ns) on more than $1 \%$ of reads. Ns occurred on $24 \%$ of R2 reads in PNUSAE013260 (Table 1) while Ns ranged from $2.6 \%$ to $3.3 \%$ on R1 reads in PNUSAE014640, PNUSAE014641, and PNUSAE014642 (Supplemental Data S4). R1 and R2 PHRED scores showed only a weak, negative association with ambiguous nucleotides $\left(r_{s}=-0.337\right.$ and -0.260$)$. On the other hand, longer reads and inserts tended to be associated with fewer overall ambiguous nucleotides $\left(r_{s}=-0.628\right.$ and -0.678$)$. WGS reads of Cluster 4 thus showed close associations between read length, insert length, and nucleotide content quality metrics.

In Cluster 4, both R1 and R2 PHRED showed significant improvements under three of the seven read healing pipelines. Prinseq raised average R2 PHRED scores to 35.3 while prinseq-3pr and prinseq-5pr3pr both raised R2 scores to 35.6 (Table 1). Under Dunn's post hoc test, prinseq, prinseq-3pr, and prinseq-5pr3pr yielded significant gains in R1 and R2 PHRED scores (Table $\mathrm{S} 5, \mathrm{df}=7$ ). Insert lengths were not significantly changed by any of the read healing pipelines (Table S5). All pipelines except noNmin100 and noNmin100-3pr significantly reduced read lengths (all $p<0.00941$ ). For instance, prinseq-5pr3pr reduced average read length by $14 \mathrm{bp}$. As with the other three outbreak clusters, decreased read lengths accompanied increased PHRED scores.

\section{Read healing and quality metrics impacting SNPs analysis.}

In Cluster 1 (E. coli), raw read quality metrics did not consistently predict read mapping as a quality metric; yet, raw reads enabled robust SNPs discovery. In Lyve-SET, mapped reads per isolate ranged from $53.2 \%$ to $76.6 \%$ (average $=66.1 \%$ ) while CFSAN SNP Pipeline mapped reads ranged from $65.6 \%$ to $82.2 \%$ (average $=74.9 \%$, Supplemental Data S1). Lyve-SET properly-paired mapped reads were lower, from $26.6 \%$ to $63.6 \%$ (average $=45.0 \%$ ) while the CFSAN SNP Pipeline did not calculate properly-paired reads. There was a strong correlation $\left(\mathrm{r}_{\mathrm{s}}\right.$ $=0.878$ ) between R2 PHRED scores and total percentage of reads mapped to the reference. R1 PHRED correlated weakly with total percentage of mapped reads $\left(r_{s}=0.232\right)$. R1 and R2 PHRED showed weak to moderate correlations with properly paired read mapping $\left(\mathrm{r}_{\mathrm{s}}=0.203\right.$ and 0.689). Read length and insert length showed negligible correlation with total mapped reads $\left(r_{s}<0.0827\right)$ while they were both moderately correlated with properly paired reads $\left(r_{s}=0.331\right.$ and 0.505 ). Mean R1 and R2 Ns percentage showed a moderate negative association with read mapping and proper pairing $\left(\mathrm{r}_{\mathrm{s}}=-0.497\right.$ and -0.487$)$. SNPs based upon raw reads yielded a multiple sequence alignment of 1,007 positions in Lyve-SET and 2,331 in CFSAN (Figure $2 \mathrm{~A}$ ). Cluster 1 raw reads recovered $97 \%$ of true positive Lyve-SET SNPs (Figure 3 A, y-axis) and $99 \%$ of true positive CFSAN SNP (Supplemental Data S5). There was a $\%$ false discovery rate for Lyve-SET (Figure 3 A, x-axis) and a $0.6 \%$ false discovery rate for CFSAN (Figure S5 A). Read-healing pipelines increased numbers of Cluster 1 SNPs detected by Lyve-SET while providing less consistent increases to CFSAN SNP. Percentage of Lyve-SET properly 
348 paired reads from all healing pipelines except noNmin 100 exhibited significant improvements 349 under the Dunn post hoc test (Figure S1 B). For instance, prinseq-3pr and prinseq-5pr3pr raised 350 properly paired mapped reads to averages around $72.4-72.5 \%$. Under prinseq- $3 \mathrm{pr}$ and prinseq$3515 \mathrm{pr} 3 \mathrm{pr}$ total mapped reads ranged from $67.4 \%$ to $80.9 \%$ in Lyve-SET and $71.0 \%$ to $84.3 \%$ in the 352 CFSAN SNP Pipeline (Supplemental Data S1). In Lyve-SET, Prinseq-3pr produced a multiple

353

354

355

356

357

358

359

360

361

362

363

364

365

366

367

368

369

370

371

372

373

374

375

376

377

378

379

380

381

382

383

384

385

386

387

sequence alignment (MSA) of 1,100 SNPs positions while Prinseq-5pr3pr yielded an MSA of 1,099 SNPs positions (Figure 2 A, Supplemental Data S5). Prinseq-3pr5pr identified 100\% of concordant Lyve-SET SNPs (True Positive Rate in Figure $3 \mathrm{~A}$ ) with only $0.2 \%$ of SNPs as predicted false discoveries (False Discovery Rate in Figure $3 \mathrm{~A}$ ). Only prinseq produced a noticeable improvement in CFSAN SNP Pipeline results, with 2,402 SNPs (Figure 2 A) and a false discovery rate near $0.4 \%$ (Figure S5 A). Healed reads in both SNP pipelines produced $98 \%$ to $100 \%$ True Positive SNPs, with $0.5 \%$ to $0.6 \%$ false discoveries (Figure 3 A; Figure S5 A; Supplemental Data S5). False discoveries averaged $0.2 \%$ for Lyve-SET and were significantly greater at $0.6 \%$ for the CFSAN SNP Pipeline (pairwise Wilcoxon, $\mathrm{p}=0.0078$ )

In Cluster 2 (S. enterica serovar Reading), raw read correlated with read mapping while the CFSAN SNP Pipeline outperformed Lyve-SET. Lyve-SET total mapped reads per isolate ranged from $54.4 \%$ to $92.8 \%$ (average $=78.5 \%$, Figure S2 A), but properly paired mapped reads were as low as $34 \%$ per isolate (average $=54.5 \%$, Figure S2 B). The CFSAN SNP Pipeline mapped reads ranged from $67.1 \%$ to $97.9 \%$ (average $=87.7 \%$, Supplemental Data S2). R2 PHRED quality scores showed robust associations with Lyve-SET total read mapping and properly paired mapping $\left(r_{s}=0.758\right.$ and 0.669$)$; R1 PHRED correlated less strongly with both $\left(r_{s}\right.$ $=0.277$ and 0.523$)$. Ns content and Lyve-SET read mapping showed a weak correlation $\left(\mathrm{r}_{\mathrm{s}}=\right.$ 0.332 ) while read length and insert length bore a negligible correlation with read mapping and properly paired mapping $\left(\mathrm{r}_{\mathrm{s}}<|-0.1844755|\right)$. In Lyve-SET, the raw reads yielded $71 \mathrm{SNPs}$ positions (Figure 2 B) while the CFSAN SNP Pipeline detected 155 SNPs (Supplemental Data S2). Lyve-SET found only $88 \%$ of estimated true positive SNPs from raw reads, yet was free from false discoveries (Figure 3 B). At the same time, the CFSAN SNP Pipeline yielded $99 \%$ of estimated true positives with a false discovery rate of $0.7 \%$ (Figure S5 B).

All seven healing pipelines improved Lyve-SET mapping and SNPs counts for Cluster 2. Read healing also contributed to robust results of the CFSAN SNP Pipeline. Reads healed through fastxOnly-3pr, noNmin100-3pr, prinseq, prinseq-3pr, and prinseq-5pr3pr exhibited significant increases in properly paired reads (Figure S2 B); Lyve-SET total mapped reads improved only slightly, averaging no more than $87.3 \%$ (Figure S2 A; Supplemental Data S2). Average total read mapping in the CFSAN SNP Pipeline reached a maximum of $90.3 \%$ for reads healed through prinseq (Supplemental Data S2). In Lyve-SET, Prinseq-5pr3pr and prinseq-3pr detected 93 to 94 SNPs positions (Figure 2 B); 100\% of these were true positives, albeit with false discovery rates of $3 \%$ (Figure $3 \mathrm{~B}$ ). In the CFSAN SNP Pipeline, prinseq-3pr, prinseq, and prinseq-5pr3pr, each detected 162, 163, and 166 SNPs, respectively. True positives of these CFSAN SNP Pipeline positions ranged from $95 \%$ to $96 \%$ with $0 \%$ false discoveries. False discoveries were statistically equivalent between the SNPs pipelines (pairwise Wilcoxon,

Peer) reviewing PDF | (2021:05:60850:1:1:NEW 10 Sep 2021) 
388

389

390

391

392

393

394

395

396

397

398

399

400

401

402

403

404

405

406

407

408

409

410

411

412

413

414

415

416

417

418

419

420

421

422

423

424

425

426

427

$\mathrm{p}=0.79$ ), with Lyve-SET averaging $0.7 \%$ and the CFSAN SNP Pipeline averaging $0.6 \%$ (Figure 3 B; Figure S5 B).

Cluster 3 (S. enterica serovar Pomona) exhibited robust correlations between raw read and SNPs quality metrics while CFSAN outperformed Lyve-SET in SNP discovery. Lyve-SET total mapped reads per isolate ranged from $41 \%$ up to $96 \%$ (average $=84.7 \%$, Figure S3 A) while properly paired reads were as low as $18 \%$ (average $=66.5 \%$, Figure S3 B). CFSAN SNP Pipeline mapped reads ranged from $87.8 \%$ to $97.7 \%$ (average $=95.6 \%$, Supplemental Data S2). R2 PHRED quality correlated strongly to mapping percentages and properly paired reads $\left(\mathrm{r}_{\mathrm{s}}=\right.$ 0.873 and 0.857 ). R1 PHRED quality was likewise strongly correlated with properly paired reads $\left(r_{s}=0.801\right)$. Read lengths and ambiguous nucleotide content exhibited weak associations with mapping of proper read pairs $\left(r_{s}=-0.173\right.$ and $\left.r_{s}=-0.298\right)$; Median insert lengths bore a moderate association with proper read pairing $\left(r_{s}=0.526\right)$. Cluster 3 raw reads aligned in LyveSET to yield 412 SNPs (Figure 2 C) while the CFSAN SNP Pipeline detected 709 SNPs. LyveSET SNPs exhibited a true positive rate of $98 \%$. The CFSAN SNP Pipeline true positives were slightly higher at $99 \%$ while both SNPs pipelines showed a $0 \%$ false discovery rate (Figure $3 \mathrm{C}$, Figure S5 C, Supplemental Data S5).

Five out of the seven healing pipelines improved SNPs analysis quality across multiple metrics for Cluster 3 . With respect to properly paired read mapping, only prinseq, prinseq$5 \mathrm{pr} 3 \mathrm{pr}$, and prinseq-3pr yielded significant improvements under the Dunn post hoc test (Table S6) where properly paired mapping rates ranged from $66 \%$ to $97 \%$ (average $=90.1 \%$ ). Prinseq increased total read mapping to similar ranges for both Lyve-SET and the CFSAN SNP Pipeline, 86.8\%-96.4\% and 88.3\%-98.0\%, respectively (Supplemental Data S3). Prinseq-3pr-healed reads yielded the largest raw number of SNPs positions in Lyve-SET, 443 in all (Figure $2 \mathrm{C}$ ), and enabled identification of $100 \%$ of the true positive positions with a $0 \%$ false discovery rate (Figure $3 \mathrm{C}$ ). All healing pipelines improved CFSAN SNP Pipeline SNPs discovery. Notably, prinseq-healed reads yielded 746 SNPs in the CFSAN SNP Pipeline (Figure 2 C) with a false discovery rate of $2.5 \%$ (Figure $3 \mathrm{C}$ ). False discoveries averaged $0.2 \%$ for Lyve-SET and $0.6 \%$ for the CFSAN SNP Pipeline, yet these rates were statistically-equivalent (pairwise Wilcoxon, $\mathrm{p}=0.42$ ).

Cluster 4 (S. sonnei) SNPs quality metrics correlated with only two of the read quality metrics, read length and insert length. In Lyve-SET, Cluster 4 isolate reads mapped to the reference from a $58.9 \%$ up to $82.5 \%$ (average $=68.9 \%$, Figure S4 A). Properly paired raw reads mapped at much lower proportions, from $45.7 \%$ to $73.1 \%$ (average $=54.4 \%$, Figure S4 B). CFSAN SNP Pipeline mapped reads ranged from $66.4 \%$ to $91.4 \%$ (average $=77.4 \%$, Supplemental Data S4). In contrast to Clusters 1-3, Cluster 4 reads exhibited little correlation between R1/R2 PHRED quality and percentage of mapped reads $\left(r_{s}=-0.210\right.$ and -0.169$)$. Average read lengths, median insert lengths, and Ns content all had negligible correlations with read mapping percentage $\left(-0.190<\mathrm{r}_{\mathrm{s}}<0.168\right)$. The Cluster 4 read lengths and insert lengths were strongly correlated with properly paired mapped reads $\left(r_{s}=0.799\right.$ and 0.775$)$. Raw reads of Cluster 4 aligning in Lyve-SET yielded 193 SNPs while the CFSAN SNP Pipeline more than 
428 tripled the number of SNPs to 608 (Figure 2 D). Both the Lyve-SET and CFSAN SNP Pipeline

429

430

431

432

433

434

435

436

437

438

439

440

441

442

443

444

445

446

447

448

449

450

451

452

453

454

455

456

457

458

459

460

461

462

463

464

465

466

showed a true positivity rate of $99 \%$ and a $0 \%$ false discovery rate (Figure $3 \mathrm{D}$ ).

For Lyve-SET in Cluster 4, all read healing pipelines except noNmin100 and bayesHammer improved SNPs analysis results; the CFSAN SNP Pipeline improved SNPs for all healed reads except noNmin 100-3pr and prinseq-3pr. FastxOnly-3pr, noNmin100-3pr, prinseq$5 \mathrm{pr} 3 \mathrm{pr}$, and prinseq-3pr improved Lyve-SET properly-paired mapping to averages of $66 \%$ to $71 \%$ (Table S6). The CFSAN SNP Pipeline performed best with prinseq read healing, yielding 629 SNP positions, and total read mapping ranging from $67.0 \%$ to $92.0 \%$ (Supplemental Data S4). Prinseq-3pr-healed reads produced 205 SNPs in Lyve-SET (Figure 2 D), a true positive rate of $99 \%$, and a false discovery rate of $2 \%$ (Figure $3 \mathrm{D}$ ). Prinseq-healed reads yielded distinct but favorable results for both Lyve-SET and the CFSAN SNP Pipeline; Lyve-SET detected 199 SNPs, a true positive rate of $99 \%$ and a false discovery rate of $0.5 \%$ (Figure $3 \mathrm{D}$ ); The CFSAN SNP Pipeline, yielded 629 SNPs, a true positive rate of $99 \%$, and a false discovery rate of $2.5 \%$ (Figure S5 D, Supplemental Data S5). False discovery rates, averaging $0.7 \%$ for Lyve-SET and $0.9 \%$ for the CFSAN SNP Pipeline, were statistically-equivalent for both SNPs pipelines (pairwise Wilcoxon, $\mathrm{p}=0.67$ ).

\section{Read healing and quality metrics relationships to assemblies.}

Raw reads from isolates in Cluster 1 (E.coli) yielded wide variations in assembly qualities while none of the read metrics correlated strongly with assembly quality. On average, raw reads assembled in SPAdes to 331 contigs, with N50 of 81,037 bp, and with maximum contig lengths around 218,070 bp (Table 2). Raw reads assembled in Skesa to an average of 341 contigs while N50 and maximum contigs averaged $75 \%$ to $80 \%$ shorter in Skesa (Table S7). Despite its high percentage of reads with ambiguous nucleotides $(\approx 4 \%), 2014 \mathrm{C}-3572$ had the best assemblies, 227 contigs in SPAdes, 221 contigs in Skesa, and near-equal maximum contigs of 296,819 in SPAdes and 296,311 in Skesa. Isolate PNUSAE001736 had the most fragmented assemblies: 435 contigs and a 48,358 bp N50 in SPAdes with 511 contigs and a 28,311 N50 in Skesa. R1 and R2 PHRED had a weak correlation with SPAdes and Skesa contig counts (-0.345 $<\mathrm{r}_{\mathrm{s}}<0.106$, (Supplemental Data S1)). Associations between average Ns per read versus contig count, N50, and maximum contig were weak $\left(-0.183<\mathrm{r}_{\mathrm{s}}<0.063\right)$. Correlations between read length, and assembly metrics were weak across SPAdes and Skesa $\left(-0.330<\mathrm{r}_{\mathrm{s}}<0.235\right)$.

Four of the seven healing pipelines enabled significant improvements in Cluster 1 assemblies. Read healing significantly improved SPAdes assemblies under the Kruskal-Wallis test; reads healed through prinseq, prinseq-3pr, prinseq-5pr3pr, and BayesHammer were significant under Dunn's post hoc test (Table 2). Prinseq-3pr also significantly improved SPAdes N50 under Dunn's post hoc test (Table 2). Read healing showed no significant improvement in Skesa assemblies (Kruskal-Wallis test, Table S7). For SPAdes assemblies, reads healed through BayesHammer assembled to the lowest (i.e. least fragmented) average contig count of 287.2 and largest maximum contig, 251,436 bp, while reads healed through

Peer) reviewing PDF | (2021:05:60850:1:1:NEW 10 Sep 2021) 
467 prinseq-3pr had the largest average N50 value at 93,141 bp (Table 2). For Skesa assemblies, 468 BayesHammer yielded the largest improvement for all three metrics: 303 contigs, 75,148 bp 469 N50, and 202,003 bp maximum contig (Table S7). Maximum contig lengths did not 470 significantly increase; yet, in both SPAdes and Skesa average maximum contigs from

471

472

473

474

475

476

477

478

479

480

481

482

483

484

485

486

487

488

489

490

491

492

493

494

495

496

497

498

499

500

501

502

503

504

505

506 BayesHammer-healed reads showed a 15\% increase over raw read assemblies (Table 2).

The raw reads generated from Cluster 2 (S. enterica serovar Reading) isolates produced assemblies of widely variable quality and were correlated with R1/R2 PHRED scores. Raw reads assembled in SPAdes to an average 96.0 contigs, a 243,104 bp N50, and 552,950 bp maximum contig lengths (Table 2). Assembly metrics for Skesa represented vast underperformance relative to SPAdes (pairwise Wilcoxon, $\mathrm{p}<0.00024$ ), showing roughly twice the average contig count as SPAdes (Table S7). Two isolates showed exceptional assemblies in SPAdes:

CVM_N17S1018, a 600-cycle sequence (Figure 1), had 36 contigs and an N50 of 455,001 bp, while PNUSAS032349, a 500-cycle sequence, exhibited the longest N50, at 637,471 bp, and the maximum contig, at 1,787,855 bp. The most robust Skesa assembly, PNUSAS032349, had 24 contigs, a 396,938 bp N50 and as the maximum contig at 1,234,155 bp (Supplemental Data S2). By comparison, APHI_17-10777 (average R2 PHRED = 27.5) had the most fragmented assembly with 266 contigs, an N50 of $28,822 \mathrm{bp}$, and a maximum contig length of 97,074. Skesa also performed poorly for APHI_17-10777, with 1,019 contigs, an N50 of 6,851 bp, and a maximum contig length of 29,736. For both SPAdes and Skesa, contig counts slightly decreased with increasing R1 and R2 PHRED scores (-0.412 $\left.<\mathrm{r}_{\mathrm{s}}<-0.437\right)$. R1 and R2 PHRED scores exhibited a moderate, positive association with N50 and maximum contig length $\left(0.430<\mathrm{r}_{\mathrm{s}}<\right.$ 0.526 ) for both assembly methods. Insert lengths and ambiguous nucleotide composition showed negligible correlation with contig count, N50, and maximum contig length $\left(-0.190<\mathrm{r}_{\mathrm{s}}<\right.$ 0.192).

Reads from isolates in Cluster 2 did not yield significant improvements in assembly quality under any of the seven healing pipelines. Under the Kruskal-Wallis test, contig counts, N50, and maximum contigs of healed read assemblies did not significantly vary from raw reads for either SPAdes (Table 2) or Skesa (Table S7). Nonetheless, reads healed through prinseq5 pr3pr showed the largest overall improvements in SPAdes assemblies; average contig count reduced to 77 (by 20\%), N50 increased by 14\%, to 277,891 bp, and average maximum contig increased by $19 \%$, to 658,148 bp (Table 2). For Skesa assemblies, BayesHammer yielded the most improvement: contig counts decreased by 34\% (to 128), N50 increased by 30\% (to 152,297 bp), and maximum contigs increased by $14 \%$ (to 366,842 bp, Table S7). Proportional improvements also occurred for prinseq-3pr, where SPAdes contig counts decreased by $20 \%$ and Skesa contig counts decreased by $28 \%$.

Cluster 3 ( $S$. enterica serovar Pomona) yielded high quality assemblies using raw reads and showed moderate associations with read quality metrics. On average, Cluster 3 raw reads assembled in SPAdes to 45 contigs, 412,213 bp N50, and 911,229 bp maximum contig lengths (Table 2). Skesa assemblies in Cluster 3 exhibited poorer assembly metrics than SPAdes (pairwise Wilcoxon, $\mathrm{p}<0.0263$ ); the average N50 and maximum contig length had only 52\% to 
$50758 \%$ the length of corresponding SPAdes metrics (Table S7). The 300-cycle sequence (Figure 508 1), 2015EL-1657B, yielded the most robust SPAdes assembly with 25 contigs and an N50 of 509757,183 , despite the occurrence of Ns on $63 \%$ of its R1 reads. The best Skesa assembly was 510 PNUSAS002461 with 21 contigs and an N50 of 455,461 bp (Supplemental Data S3).

511 PNUSAS002458, with an R1 PHRED score of 29.8 and 10\% Ns on its R2 reads, yielded the 512 poorest SPAdes assembly, 151 contigs and an N50 of 220,687 bp. Through Skesa,

513 PNUSAS002458 yielded 91 contigs and an N50 of 96,273 bp. The correlations between

514 ambiguous nucleotides and contig count, N50, and maximum contig length were negligible ($\left.5150.0531<\mathrm{r}_{\mathrm{s}}<0.174\right)$. R1 and R2 PHRED showed a moderate inverse association with contig 516 counts for SPAdes and Skesa $\left(-0.493>r_{s}>-0.301\right)$. SPAdes/Skesa N50 and maximum contig 517 length increased moderately with R1 and R2 PHRED $\left(0.351<\mathrm{r}_{\mathrm{s}}<0.591\right)$. Insert lengths also 518 increased with maximum contig lengths $\left(\mathrm{r}_{\mathrm{s}}=0.5354\right)$. Otherwise, insert lengths and read lengths 519 showed little association with contig counts or N50 $\left(-0.3984<\mathrm{r}_{\mathrm{s}}<0.2554\right)$.

520

Read-healing pipelines made only slight improvements to assembly quality metrics for

521 Cluster 3. Read healing did not significantly change contig count, N50, or maximum contig 522 length for either SPAdes (Table 2) or Skesa (Table S7) under the Kruskal-Wallis test. Despite 523 the absence of statistically robust improvements, prinseq-5pr3pr, prinseq-3pr, and BayesHammer 524 provided proportional gains to assembly quality metrics. Prinseq-5pr3pr reduced average 525 SPAdes and Skesa contig counts to 35 and 42, respectively; these comprised $20 \%$ and $15 \%$ 526 reductions from the average raw read contig counts (Table 2 and Table S7). Prinseq-3pr

527

528

529

530

531

532

533

534

535

536

537

538

539

540

541

542

543

544

545

546 increased SPAdes N50 to an average of 465,935 bp (a 13\% increase, Table 2) and Prinseq increased Skesa maximum contig length to 558,596 bp (a 16\% increase, Table S7).

Cluster 4 ( $S$. sonnei) assemblies were considerably more fragmented than those of Clusters 1 through 3. On average, Cluster 4 raw reads in SPAdes assembled to 449.8 contigs, a 23,931 bp N50, and 89,893 bp maximum contig length (Table 2). Skesa assembled Cluster 4 into fewer contigs, 392 on average, significantly less fragmented than SPAdes (pairwise Wilcoxon, $p=0.0000317$ ). At the same time, Skesa produced an average 23,494 bp N50 and an 88,610 bp maximum contig length (Table S7). Isolate PNUSAE013260 yielded the most fragmented SPAdes assembly with 501 contigs and an N50 of 19,019 bp; the Skesa assembly was somewhat less fragmented with 468 contigs and an N50 of 18,211 bp. Isolate PNUSAE013900 exhibited the best Cluster 4 assembly, with SPAdes yielding 408 contigs and a 25,378 bp N50; Skesa yielded 359 contigs and a 25,647 bp N50. For both assemblers, R1 and R2 PHRED scores bore only weak to moderate correlations with contig count, N50, or maximum contig length $\left(-0.4144<\mathrm{r}_{\mathrm{s}}<0.2166\right.$, Supplemental Data S4). For SPAdes assemblies, maximum contig length showed an unexpected decreasing trend with read length and insert length $\left(\mathrm{r}_{\mathrm{s}}=\right.$ 0.6061 and -0.5866). For Skesa, maximum contig length also showed an unexpected increase with Ns content $\left(r_{s}=0.506\right)$. Read length and insert length showed only weak correlations with contig count and N50 $\left(-0.384<\mathrm{r}_{\mathrm{s}}<0.2462\right)$.

None of the read healing pipelines significantly improved any of the assembly metrics for Cluster 4 reads. Under the Kruskal-Wallis test, neither contig count, N50, nor maximum contig 
547 length could be improved by read healing by either SPAdes (Table 2) or Skesa (Table S7). A

548 few proportional improvements were observed. BayesHammer-healed reads assembled to an

549 average of 443.4 contigs in SPAdes and 386 contigs in Skesa; both represented a less than 2\%

550 decrease from raw read contig counts (Table 2). For SPAdes, prinseq-healed reads assembled to

551 an average N50 of 24,117 bp (less than a 1\% improvement, Table 2). Skesa lengthened average

552 maximum contigs from prinseq-healed reads by 1\%; At the same time, metrics did not

553 consistently improve across Skesa assemblies of healed reads (Table S7).

\section{Discussion}

555

556

557

558

559

560

561

562

563

564

565

566

567

568

569

570

571

572

573

574

575

576

577

578

579

580

581

582

583

584

585

This study has shown that WGS quality metrics, particularly PHRED scores, have varying impacts upon analyses of bacterial genomes with average $\mathrm{G}+\mathrm{C}$ content. As of August 2020, Illumina data at NCBI SRA (https://www.ncbi.nlm.nih.gov/sra) were available for 198,928 E. coli, 343,457 S. enterica, and 13,374 S. sonnei isolates. Given this volume of WGS data representing a wide variation in quality control standards, methods of defining and improving WGS quality are needed.

R1 and R2 PHRED scores exerted the strongest effects on SNPs analyses while insert length and read length effects on SNPs could not be ruled out. R1 and R2 PHRED scores exhibited positive correlations with the mapping stage of SNPs analyses in all except Cluster 4. Two of the three PHRED-quality-trimming-based healing methods, prinseq and prinseq-3, yielded enhanced SNPs discovery results in the CFSAN SNP Pipeline and Lyve-SET, respectively. High PHRED scores have previously been shown to increase proportions of mapped reads, which in turn increased numbers of SNPs discovered (Yu et al. 2012; Yu \& Sun 2013). By contrast, ambiguous nucleotide composition (Ns) for raw reads showed only a slight association with read mapping in Cluster 1 and none in Clusters 2 through 4. Moreover, the ambiguity-free reads processed by noNmin 100 underperformed in SNPs identification relative to all other healed sets. Hence, ambiguous nucleotide composition is not a critical quality metric for Illumina WGS data of enteric bacteria. Insert length showed a positive association with properly-paired mapped reads in Cluster 4, and to a lesser extent in Clusters 1 and 3. Greater proportions of reads mapping in proper pairs, in turn, may have enabled identification of more SNPs. Read length was positively correlated with insert lengths among 500-cycle reads across the four clusters. The strong association between read lengths and insert lengths in Cluster 4 make it unclear which two quality metrics impacted SNPs analysis the most. Despite previous studies (Chen-Harris et al. 2013; Guan \& Sung 2016; Laehnemann et al. 2016) and this current study, further effort may be needed to show how insert sizes affect SNPs and how insert length, read length, and PHRED scores affect number and quality of SNPs.

Choice of read-healing strategy also impacted SNPs analyses. Five of the read healing pipelines, fastxOnly-3pr, noNmin 100-3pr, prinseq, prinseq-5pr3pr, and prinseq-3pr, all employed trimming of 3' and/or 5' ends of reads. By contrast, noNmin 100 employed filtering only and BayesHammer employed correction only. NoNmin100 filtering (no trimming) performed well for Lyve-SET in Cluster 2, but underperformed for the other clusters with respect

PeerJ reviewing PDF | (2021:05:60850:1:1:NEW 10 Sep 2021) 
586 to SNPs counts and/or true positive rate. The simple, blunt-end trimming, fastxOnly-3pr, 587 underperformed for the CFSAN SNP Pipeline. Prinseq, which trimmed based upon PHRED 588 quality, proved to be effective for increasing SNPs counts for both Lyve-SET and the CFSAN 589 SNP Pipeline. Trimming was been shown to increase concordant (estimated true positive) SNPs 590 in a study for human genome clinical Illumina data (Yu \& Sun 2013). At the same time, another 591 study showed trimming to decrease numbers of true positive SNPs, albeit by only $0.7 \%$ (Liu et 592 al. 2012). In our study, prinseq-healed reads enabled detection of a greater or equal amount of 593 true positive SNPs compared to raw reads across all clusters except for Cluster 2 in the CFSAN 594 SNP Pipeline. False discovery rates in our study reached a maximum of $3 \%$ for reads healed 595 through prinseq-5pr3pr and prinseq-3pr (Cluster 2 only, Figure 3 B). These two healing methods 596 produced less than $1.5 \%$ false discoveries in the other three clusters for both SNP pipelines. Indeed, prinseq-5pr3pr and prinseq-3pr yielded less than $1 \%$ false discoveries for the CFSAN SNP Pipeline. Such increases in SNPs analysis performance matches the significant increase in PHRED scores provided by prinseq-5pr3pr and prinseq-3pr.

600

601

The CFSAN SNP Pipeline detected larger numbers of SNPs relative to Lyve-SET, possibly due to the former's less stringent coverage threshold of $8 \mathrm{X}$ (versus 20X in Lyve-SET).

602

603 The CFSAN SNP Pipeline also mapped higher proportions of reads, as it employs Bowtie2 (Langmead \& Salzberg 2012), compared with the more stringent SMALT in Lyve-SET (Katz et al. 2017; Ponstingl \& Ning 2010). Given that Lyve-SET is designed to be more conservative, it is surprising that Lyve-SET and the CFSAN SNP Pipeline produced similar proportions of false discoveries for three out of the four clusters. In any case, the quality-based trimming methods, prinseq, prinseq-5pr3pr, and prinseq-3pr, plus the correction-based BayesHammer, enhanced

609 SNPs analysis across both SNPs pipelines. Accuracy of SNPs detection may depend less upon choice of SNPs pipeline and more upon choice of read-healing and the peculiarities of the microbial cluster under consideration.

Contrasting with SNPs analysis quality, associations between read quality metrics and assembly quality were less consistent. R1 and R2 PHRED scores did show some association with reduced contig counts in the Salmonella clusters ( 2 and 3). The three quality-based trimming methods yielded significant improvements in Cluster 1 only. As was the case for SNPs,

616 Unexpectedly, insert lengths were not strongly associated with robust assemblies; greater insert

617 lengths correlated with maximum contig lengths in Cluster 3 only. A previous study suggested

618 that higher insert average length and more variable insert lengths lead to less fragmented

619 assemblies (Huptas 2016). Yet, the variability in read length chemistry in the current study may 620 have obscured any relationship between insert length, read length and assembly metrics. While

621 PHRED scores positively impacted assembly quality, additional read quality metrics, not

622 measured by this study, may also be relevant. Previous studies have shown genomic GC content

623 bias (Chen et al. 2013; Huptas 2016; Ross et al. 2013) and Illumina-specific substitution errors

624 (Laehnemann et al. 2016) to impact genome assemblies. Cluster 4 assemblies exhibited

625 seemingly intractable levels of fragmentation, never more contiguous than 354 contigs (i.e., 
626 Skesa after BayesHammer-healing). Cluster 4 represents S. sonnei, which exhibit highly627 fragmented assemblies. The current study represents a modest improvement over Shigella 628 assembly averages around 500 contigs in a previous study (Timme et al. 2018). The high 629 incidence of insertion sequences, repeats, and recent rearrangements in Shigella spp. genomes 630 likely make assembly of these organisms more challenging than other enteric bacteria (Page et 631 al. 2016; Yang et al. 2005). Further investigation into optimizing read quality metrics for

632 633 634 635

636

637

638

639

640

641

642

643

644

645

646

647

648

649

650

651

652

653

654

655

656

657

658

659

660

661

662

663

664

665 improved draft genome assemblies of Shigella spp. and other enterics may be needed.

The findings of this study are subject to several limitations given that it used real WGS data rather than simulated data. First, all the Illumina reads in this study were obtained via Nextera XT library kits (Quail et al. 2012; Syed et al. 2009). Nextera XT libraries have welldocumented quality issues, particularly $\mathrm{G}+\mathrm{C} \%$ bias (Sato et al. 2019; Tyler et al. 2016). The KAPA and TruSeq have been shown to produce higher quality bacterial genomic reads with less fragmentation of genome assemblies (Jones et al. 2015; Seth-Smith et al. 2019). Compared to Nextera XT, Nextera DNA Prep reads also assembled to higher quality draft genomes as measured by N50, contig count, and plasmid detection (Haendiges et al. 2021). For this study, reads from such higher-quality libraries would likely have behaved differently under the healing pipelines investigated. Second, Clusters 1 through 3 exhibited mixed Illumina sequencing chemistries, with small numbers of 300-cycle and 600-cycle analyzed along with the majority 500-cycle. The 151-bp 300-cycle reads in these clusters tended to have higher PHRED quality than 500-cycle or 600-cycle. By contrast, the 301-bp 600-cycle reads trended towards the lowest PHRED quality scores. While these trends in read cycle chemistry are not generalizable to other studies, they made analyses in this study more complex. Third, the choice of outbreak clusters in this study was restricted to enteric bacteria with neutral GC content (50\% to $55 \%)$. Results for read and analysis quality metrics from this study may not be relevant to low-GC foodborne pathogens such as Listeria monocytogenes and Campylobacter spp. Fourth, the scope of quality metrics analyzed in this study did not include substitution nor indel errors for the Illumina reads. Previous investigations into Illumina sequencing quality found that PHRED scores are not always a reliable predictor of substitution/indel errors (Schirmer et al. 2016; Zhang et al. 2017). Future studies of enteric bacterial WGS quality may need to incorporate quality score recalibration into SNPs analysis to compensate for WGS errors and biases (DePristo et al. 2011; O'Rawe et al. 2013). Further SNPs validation of enteric bacteria would benefit greatly by incorporating a cluster of microbial genomes with validated or curated SNPs. Finally, assemblies in this study were measured only for contiguity, not correctness. A simulated read set derived from a finished genome could have provided metrics for assembly correctness, as employed in the Assemblathon (Earl et al. 2011). On the other hand, simulated data may not represent the errors or variation present in real WGS reads (Salzberg et al. 2012). While SPAdes has been demonstrated to be among the most accurate assemblers for small genomes (Huptas 2016), Skesa showed favorable results in assemblies of S. sonnei (Cluster 4). Hence, limitations to this study are related to the chosen WGS data sets in this study: enteric bacteria linked to outbreaks. 
666

\section{Conclusions}

668 WGS has greatly enriched evolutionary and molecular studies of enteric bacteria while

669 expediting outbreak responses to these common and widespread pathogens. As WGS grows in

670 importance to clinical bacteriology, additional computational methods, such as MLST, for

671 outbreak prediction will develop further (Besser et al. 2018; Besser et al. 2019; Moran-Gilad et

672 al. 2015; Page et al. 2017). Moreover, metagenomics approaches to WGS may become

673 indispensable as culture-independent diagnostics of enteric infections becomes widespread

674 (Besser et al. 2018). Future studies should elucidate WGS quality metrics effects upon MLST

675 and metagenomics. In this study, multiple read-healing methods performed well for SNPs analysis; choice of read-healing method was shown to be more important than choice of SNP

677 pipeline. However, the read-healing methods detailed in this study had less impact upon genome

678 assembly. Thus, read-healing methods which improve SNPs analyses may or may not be

679 effective for MLST or metagenomics. In future studies, MLST, metagenomics, or other WGS

680 analyses may require customized preprocessing tools to yield optimal results. As an additional

681 future study, misassemblies of genomes could be evaluated with respect to read quality metrics.

682 Regardless of the specifics of WGS development, quality metrics such as PHRED score, read

683 length, insert length, and various nucleotide compositional measurements will allow comparison

684 between datasets and methodologies. As WGS capabilities expand, standards for these quality

685 metrics must be evaluated and adapted for all stakeholders.

686

687

688

689

690

691

692

693

694

695

696

697

698

699

700

\section{Acknowledgements}

We thank Soledad Ulloa Urrutia and Fernández Ordenes of Instituto de Salud Pública de Chile, Tonya Mackie and Tod Stuber of the Animal and Plant Health Inspection Service, USDA, and the Enteric Diseases Laboratory Branch, CDC, for contributing WGS data for the study.

\section{References}

Arndt D, Grant JR, Marcu A, Sajed T, Pon A, Liang Y, and Wishart DS. 2016. PHASTER: a better, faster version of the PHAST phage search tool. Nucleic Acids Research 44:W16W21.

Bankevich A, Nurk S, Antipov D, Gurevich AA, Dvorkin M, Kulikov AS, Lesin VM, Nikolenko SI, Pham S, Prjibelski AD, Pyshkin AV, Sirotkin AV, Vyahhi N, Tesler G, Alekseyev MA, and Pevzner PA. 2012. SPAdes: A new genome assembly algorithm and Its applications to single-cell sequencing. Journal of Computational Biology 19:455-477. 
701

702

703

704

705

706

707

708

709

710

711

712

713

714

715

716

717

718

719

720

721

722

723

724

725

726

727

728

729

730

731

732

733

734

735

736

737

738

739

740

741

742

743

744

745

746

747

748

749

750

Besser J, Carleton HA, Gerner-Smidt P, Lindsey RL, and Trees E. 2018. Next generation sequencing technologies and their application to the study and control of bacterial infections. Clinical Microbiology and Infection 24:335-341.

Besser JM, Carleton HA, Trees E, Stroika SG, Hise K, Wise M, and Gerner-Smidt P. 2019. Interpretation of whole-genome sequencing for enteric disease surveillance and outbreak investigation. Foodborne Pathogens and Disease 16:504-512.

Cheleuitte-Nieves C, Gulvik CA, McQuiston JR, Humrighouse BW, Bell ME, Villarma A, Fischetti VA, Westblade LF, and Lipman NS. 2018. Genotypic differences between strains of the opportunistic pathogen Corynebacterium bovis isolated from humans, cows, and rodents. PLOS ONE 13.

Chen-Harris H, Borucki MK, Torres C, Slezak TR, and Allen J. 2013. Ultra-deep mutant spectrum profiling: improving sequencing accuracy using overlapping read pairs. BMC Genomics 14.

Chen T-W, Gan R-C, Chang Y-F, Liao W-C, Wu TH, Lee C-C, Huang P-J, Lee C-Y, M. CY-Y, Chiu C-H, and Tang P. 2015. Is the whole greater than the sum of its parts? De novo assembly strategies for bacterial genomes based on paired-end sequencing. BMC Genomics 16:648.

Chen Y-C, Liu T, Yu C-H, Chiang T-Y, and Hwang C-C. 2013. Effects of GC bias in nextgeneration-sequencing data on De Novo genome assembly. PLOS ONE 8.

Clooney AG, Fouhy F, Sleator RD, O' Driscoll A, Stanton C, Cotter PD, and Claesson MJ. 2016. Comparing apples and oranges?: next generation sequencing and its impact on microbiome analysis. PLOS ONE 11.

Crowe SJ, Bottichio L, Shade LN, Whitney BM, Corral N, Melius B, Arends KD, Donovan D, Stone J, Allen K, Rosner J, Beal J, Whitlock L, Blackstock A, Wetherington J, Newberry LA, Schroeder MN, Wagner D, Trees E, Viazis S, Wise ME, and Neil KP. 2017. Shiga toxin-producing E. coli infections associated with flour. The New England Journal of Medicine 377:2036-2043.

Davis S, Pettengill JB, Luo Y, Payne J, Shpuntoff A, Rand H, and Strain E. 2015. CFSAN SNP Pipeline: an automated method for constructing SNP matrices fromnext-generation sequence data. PeerJ Computer Science 1. 10.7717/peerj-cs.20

DePristo MA, Banks E, Poplin RE, Garimella KV, Maguire JR, Hartl C, Philippakis AA, del Angel G, Rivas MA, Hanna M, McKenna A, Fennell TJ, Kernytsky AM, Sivachenko AY, Cibulskis K, Gabriel SB, Altshuler D, and Daly MJ. 2011. A framework for variation discovery and genotyping using next-generation DNA sequencing data. Nature Genetics 43:491-498.

Dohm JC, Lottaz C, Borodina T, and Himmelbauer H. 2008. Substantial biases in ultra-short read data sets from high-throughput DNA sequencing. Nucleic Acids Research 36.

Earl D, Bradnam K, St. John J, Darling A, Lin D, Fass J, Yu HOK, Buffalo V, Zerbino DR, Diekhans M, Nguyen N, Ariyaratne PN, Sung W-K, Zemin Ning, Matthias Haimel, Jared T. Simpson, Nuno A. Fonseca, Inanç Birol, T. Roderick Docking, Isaac Y. Ho, Daniel S. Rokhsar, Rayan Chikhi, Dominique Lavenier, Guillaume Chapuis, Delphine Naquin, Nicolas Maillet, Michael C. Schatz, David R. Kelley, Adam M. Phillippy, Sergey Koren, Shiaw-Pyng Yang, Wei Wu, Wen-Chi Chou, Anuj Srivastava, Timothy I. Shaw, J. Graham Ruby, Peter Skewes-Cox, Miguel Betegon, Michelle T. Dimon, Victor Solovyev, Igor Seledtsov, Petr Kosarev, Denis Vorobyev, Ricardo Ramirez-Gonzalez, Richard Leggett, Dan MacLean, Fangfang Xia, Ruibang Luo, Zhenyu Li, Yinlong Xie, Binghang Liu, Sante Gnerre, lain MacCallum, Dariusz Przybylski, Filipe J. Ribeiro, Shuangye Yin, Ted Sharpe, Giles Hall, Paul J. Kersey, Richard Durbin, Shaun D. Jackman, Jarrod A. Chapman, Xiaoqiu Huang, Joseph L. DeRisi, Mario Caccamo, Yingrui Li, David B. Jaffe, Richard E. Green, David Haussler, lan Korf, and Paten B. 2011. Assemblathon 1: A

Peer) reviewing PDF | (2021:05:60850:1:1:NEW 10 Sep 2021) 
751

752

753

754

755

756

757

758

759

760

761

762

763

764

765

766

767

768

769

770

771

772

773

774

775

776

777

778

779

780

781

782

783

784

785

786

787

788

789

790

791

792

793

794

795

796

797

798

799

800

801

competitive assessment of de novo short read assembly methods. Genome Research 21:2224-2241.

Ewing B, and Green P. 1998. Base-calling of automated sequencer traces using Phred. II. error probabilities. Genome Research 8:186-194.

Fabbro CD, Scalabrin S, Morgante M, and Giorgi FM. 2013. An extensive evaluation of read trimming effects on Illumina NGS data analysis. PLOS ONE 8.

Gambino-Shirley K, Stevenson L, Concepción-Acevedo J, Trees E, Wagner D, Whitlock L, Roberts J, Garrett N, Van Duyne S, McAllister G, Schick B, Schlater L, Peralta V, Reporter R, Li L, Waechter H, Gomez T, Fernández Ordenes J, Ulloa S, Ragimbeau C, Mossong J, and Nichols M. 2018. Flea market finds and global exports: Four multistate outbreaks of human Salmonella infections linked to small turtles, United States--2015. Zoonoses Public Health 65:560-568.

Goris J, Konstantinidis KT, Klappenbach JA, Coenye T, Vandamme P, and Tiedje JM. 2007. DNA-DNA hybridization values and their relationship to whole-genome sequence similarities. International Journal of Systematic and Evolutionary Microbiology 57:81-91.

Guan P, and Sung W-K. 2016. Structural variation detection using next-generation sequencing data a comparative technical review. Methods 102:36-49.

Guo Y, Li J, Li C-I, Long J, Samuels DC, and Shyr Y. 2012. The effect of strand bias in Illumina short-read sequencing data. BMC Genomics 13.

Haendiges J, Jinneman K, and Gonzalez-Escalona N. 2021. Choice of library preparation affects sequence quality, genome assembly, and precise in silico prediction of virulence genes in shiga toxin producing Escherichia coli. PLOS ONE 16. 10.1371/journal.pone.0242294

Hannon GJ. 2010. FASTX-Toolkit. Available at http://hannonlab.cshl.edu/fastx toolkit/. Head SR, Komori HK, LaMere SA, Whisenant T, Nieuwerburgh FV, Salomon DR, and Ordoukhanian P. 2014. Library construction for next-generation sequencing: overviews and challenges. Biotechniques 56.

Heydari M, Miclotte G, Demeester P, Van de Peer Y, and Fostier J. 2017. Evaluation of the impact of Illumina error correction tools on de novo genome assembly. BMC Bioinformatics 18:374.

Huptas C, Scherer, Siegfried, Wenning, Mareike. 2016. Optimized Illumina PCR-free library preparation for bacterial whole genome sequencing and analysis of factors influencing de novo assembly. BMC Research Notes 9:269.

Jones MB, Highlander SK, Anderson EL, Li W, Dayrit M, Klitgord N, Fabani MM, Seguritan V, Green J, Pride DT, Yooseph S, Biggs W, Nelson KE, and Venter JC. 2015. Library preparation methodology can influence genomic and functional predictions in human microbiome research. Proceedings of the National Academy of Sciences 112:1402414029.

Katz LS, Griswold T, Williams-Newkirk AJ, Wagner D, Petkau A, Sieffert C, Domselaar GV, Deng X, and Carleton HA. 2017. A comparative analysis of the Lyve-SET phylogenomics pipeline for genomic epidemiology of foodborne pathogens. Frontiers in Microbiology 8.

Katz LS, Petkau A, Beaulaurier J, Tyler S, Antonova ES, Turnsek MA, Guo Y, Wang S, Paxinos EE, Orata F, Gladney LM, Stroika S, Folster JP, Rowe L, Freeman MM, Knox N, Frace M, Boncy J, Graham M, Hammer BK, Boucher Y, Bashir A, and Hanage WP. 2013. Evolutionary dynamics of Vibrio cholerae $\mathrm{O} 1$ following a single-source introduction to Haiti. mBio 4:e00398-00313.

Kelley DR, Schatz MC, and Salzberg SL. 2010. Quake: quality-aware detection and correction of sequencing errors. Genome Biology 11.

Kislyuk AO, Katz LS, Agrawal S, Hagen MS, Conley AB, Jayaraman P, Nelakuditi V, Humphrey JC, Sammons SA, Govil D, Mair RD, Tatti KM, Tondella ML, Harcourt BH, Mayer LW,

Peer) reviewing PDF | (2021:05:60850:1:1:NEW 10 Sep 2021) 
802

803

804

805

806

807

808

809

810

811

812

813

814

815

816

817

818

819

820

821

822

823

824

825

826

827

828

829

830

831

832

833

834

835

836

837

838

839

840

841

842

843

844

845

846

847

848

849

850

851

and Jordan IK. 2010. A computational genomics pipeline for prokaryotic sequencing projects. Bioinformatics 26:1819-1826.

Laehnemann D, Borkhardt A, and Carolyn McHardy A. 2016. Denoising DNA deep sequencing data-high-throughput sequencing errors and their correction. Briefings in Bioinformatics 17:154-179.

Langmead B, and Salzberg S. 2012. Fast gapped-read alignment with Bowtie 2. Nature Methods 9:357-359.

Li H, Handsaker B, Wysoker A, Fennell T, Ruan J, Homer N, Marth G, Abecasis G, Durbin R, and Subgroup GPDP. 2009. The sequence alignment/map format and SAMtools. Bioinformatics 25:2078-2079.

Liu Q, Guo Y, Li J, Long J, Zhang B, and Shyr Y. 2012. Steps to ensure accuracy in genotype and SNP calling from Illumina sequencing data. BMC Genomics 13.

Marshall KEH, Tewell M, Tecle S, Leeper M, Sinatra J, Kissler B, Fung A, Brown K, Wagner D, Trees E, Hise KB, Chaturvedi V, Schlater LK, Morningstar-Shaw BR, Whitlock L, Holt K, Becker K, Nichols M, Williams IT, Jhung M, Wise ME, and Gieraltowski L. 2018. Protracted outbreak of Salmonella Newport infections linked to ground beef: Possible role of dairy cows - 21 states, 2016-2017. Morbidity and Mortality Weekly Report 67:443-446.

Minoche AE, Dohm JC, and Himmelbauer H. 2011. Evaluation of genomic high-throughput sequencing data generated on Illumina HiSeq and Genome Analyzer systems. Genome Biology 12.

Moran-Gilad J, Sintchenko V, Pedersen SK, Wolfgang WJ, Pettengill J, Strain E, Hendriksen RS, and 4 GMlisWG. 2015. Proficiency testing for bacterial whole genome sequencing: an end-user survey of current capabilities, requirements and priorities. BMC Infectious Diseases 15.

Nikolenko SI, Korobeynikov Al, and Alekseyev MA. 2013. BayesHammer: Bayesian clustering for error correction in single-cell sequencing. BMC Genomics 14.

O'Rawe J, Jiang T, Sun G, Wu Y, Wang W, Hu J, Bodily P, Tian L, Hakonarson H, Johnson WE, Wei Z, Wang K, and Lyon GJ. 2013. Low concordance of multiple variant-calling pipelines: practical implications for exome and genome sequencing. Genome Medicine 5.

Page AJ, Alikhan N-F, Carleton HA, Seemann T, Keane JA, and Katz LS. 2017. Comparison of classical multi-locus sequence typing software for next-generation sequencing data. Microbial Genomics 3.

Page AJ, Silva ND, Hunt M, Quail MA, Parkhill J, Harris SR, Otto TD, and Keane JA. 2016. Robust high-throughput prokaryote de novo assembly improvement pipeline for Illumina data. Microbial Genomics 2:e000083. 10.1099/mgen.0.000083

Patel RK, and Jain M. 2012. NGS QC Toolkit: A Toolkit for Quality Control of Next Generation Sequencing Data. PLOS ONE 7.

Ponstingl $\mathrm{H}$, and Ning Z. 2010. SMALT - A new mapper for DNA sequencing reads. F1000Posters. Intelligent Systems for Molecular Biology 2010 meeting.

Quail MA, Smith M, Coupland P, Otto TD, Harris SR, Connor TR, Bertoni A, Swerdlow HP, and Gu Y. 2012. A tale of three next generation sequencing platforms: comparison of lon Torrent, Pacific Biosciences and Illumina MiSeq sequencers. BMC Genomics 13:341.

Ross MG, Russ C, Costello M, Hollinger A, Lennon NJ, Hegarty R, Nusbaum C, and Jaffe DB. 2013. Characterizing and measuring bias in sequence data. Genome Biology 14.

Salzberg SL, Phillippy AM, Zimin A, Puiu D, Magoc T, Koren S, Treangen TJ, Schatz MC, Delcher AL, Roberts M, Marcxais G, Pop M, and Yorke JA. 2012. GAGE: A critical evaluation of genome assemblies and assembly algorithms. Genome Research 22:557567.

PeerJ reviewing PDF | (2021:05:60850:1:1:NEW 10 Sep 2021) 
852

853

854

855

856

857

858

859

860

861

862

863

864

865

866

867

868

869

870

871

872

873

874

875

876

877

878

879

880

881

882

883

884

885

886

887

888

889

890

891

892

893

894

895

896

897

898

899

900

901

902

Sato MP, Ogura Y, Nakamura K, Nishida R, Gotoh Y, Hayashi M, Hisatsune J, Sugai M, Takehiko I, and Hayashi T. 2019. Comparison of the sequencing bias of currently available library preparation kits for Illumina sequencing of bacterial genomes and metagenomes. DNA Research 26:391-398.

Schirmer M, D'Amore R, ljaz UZ, Hall N, and Quince C. 2016. Illumina error profiles: resolving fine-scale variation in metagenomic sequencing data. BMC Bioinformatics 17:125.

Schmieder R, and Edwards R. 2011. Quality control and preprocessing of metagenomic datasets. Bioinformatics 27:863-864.

Seth-Smith HMB, Bonfiglio F, Cuenod A, Reist J, Egli A, and Wuthrich D. 2019. Evaluation of Rapid Library Preparation Protocols for Whole Genome Sequencing Based Outbreak Investigation. Front Public Health 7:241. 10.3389/fpubh.2019.00241

Singh NK, Bezdan D, Sielaff AC, Wheeler K, Mason CE, and Venkateswaran K. 2018. Multidrug resistant Enterobacter bugandensis species isolated from the International Space Station and comparative genomic analyses with human pathogenic strains $B M C$ Microbiology 18.

Smeds L, and Künstner A. 2011. CONDETRI - A content dependent read trimmer for Illumina data. PLOS ONE 6.

Souvorov A, Agarwala R, and Lipman DJ. 2018. SKESA: strategic k-mer extension for scrupulous assemblies. Genome Biology 19. s13059-018-1540-z

Syed F, Grunenwald H, and Caruccio N. 2009. Next-generation sequencing library preparation: simultaneous fragmentation and tagging using in vitro transposition. Nature Methods 6. https://doi.org/10.1038/nmeth.f.272

Tan G, Opitz L, Schlapbach R, and Rehrauer H. 2019. Long fragments achieve lower base quality in Illumina paired-end sequencing. Scientific Reports 9.

Timme RE, Rand H, Leon MS, Hoffmann M, Strain E, Allard M, Roberson D, and Baugher JD. 2018. GenomeTrakr proficiency testing for foodborne pathogen surveillance: an exercise from 2015. Microbial Genomics 4.

Trivedi UH, Cézard T, Bridgett S, Montazam A, Nichols J, Blaxter M, and Gharbi K. 2014. Quality control of next-generation sequencing data without a reference. Frontiers in Genetics 5.

Turner FS. 2014. Assessment of insert sizes and adapter content in fastq data from NexteraXT libraries. Frontiers in Genetics 5.

Tyler AD, Christianson S, Knox NC, Mabon P, Wolfe J, Van Domselaar G, Graham MR, and Sharma MK. 2016. Comparison of sample preparation methods used for the nextgeneration sequencing of Mycobacterium tuberculosis. PLOS ONE 11:e0148676.

Walters MS, Simmons L, Anderson TC, DeMent J, Van Zile K, Matthias LP, Etheridge S, Baker R, Healan C, Bagby R, Reporter R, Kimura A, Harrison C, Ajileye K, Borders J, Crocker K, Smee A, Adams-Cameron M, Joseph LA, Tolar B, Trees E, Sabol A, Garrett N, Bopp C, Bosch S, and Behravesh CB. 2016. Outbreaks of salmonellosis from small turtles. Pediatrics 137.

Wang H, Wang J, Yu P, Ge P, Jiang Y, Xu R, Chen R, and Liu X. 2017. Identification of antibiotic resistance genes in the multidrug-resistant Acinetobacter baumannii strain, MDR-SHH02, using whole-genome sequencing. International Journal of Molecular Medicine 39.

Whiteford N, Skelly T, Curtis C, Ritchie M, Lohr A, Zaranek AW, Abnizova I, and Brown C. 2009. Swift: primary data analysis for the Illumina Solexa sequencing platform. Bioinformatics 25:2194-2199.

Wood DE, and Salzberg SL. 2014. Kraken: ultrafast metagenomic sequence classification using exact alignments. Genome Biology 15. 10.1186/gb-2014-15-3-r46

Yang $F$, Yang J, Zhang X, Chen L, Jiang Y, Yan Y, Tang X, Wang J, Xiong Z, Dong J, Xue Y, Zhu Y, Xu X, Sun L, Chen S, Nie H, Peng J, Xu J, Wang Y, Yuan Z, Wen Y, Yao Z,

Peer) reviewing PDF | (2021:05:60850:1:1:NEW 10 Sep 2021) 
903

904

905

906

907

908

909

910

911

912

913

914
Shen Y, Qiang B, Hou Y, and Yu J. 2005. Genome dynamics and diversity of Shigella species, the etiologic agents of bacillary dysentery. Nucleic Acids Research 33:64456458.

Yu X, Guda K, Willis J, Veigl M, Wang Z, Markowitz S, Adams MD, and Sun S. 2012. How do alignment programs perform on sequencing data with varying qualities and from repetitive regions? BioData Mining 5.

Yu X, and Sun S. 2013. Comparing a few SNP calling algorithms using low-coverage sequencing data. BMC Bioinformatics 14.

Zhang S, Wang B, Wan L, and Lei ML. 2017. Estimating Phred scores of Illumina base calls by logistic regression and sparse modeling. BMC Bioinformatics 18:335. 


\section{Figure 1}

Illumina Read Chemistries Used in the Study.

Forward reads (R1, in blue) and reverse reads ( $R 2$, red) with range of lengths and average lengths found in raw reads. Insert sizes were inferred from SMALT mapping to draft genome assemblies and are given here as per isolate median lengths between 5' position of R1 and the 5' position of R2.

\section{0-cycle}

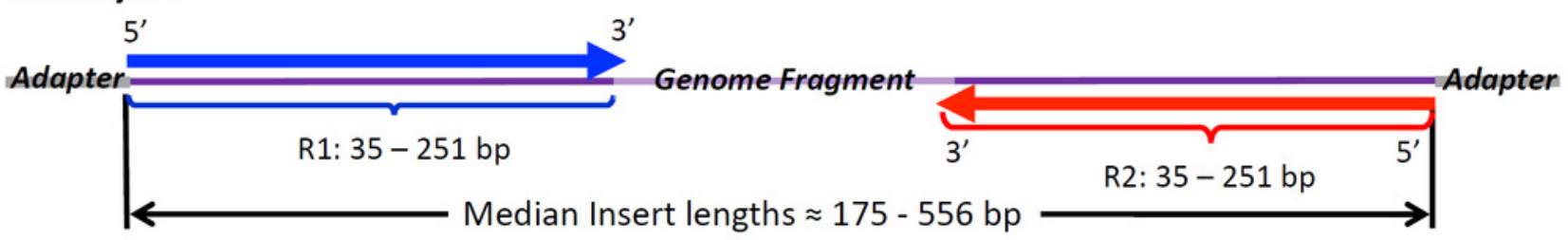

600-cycle

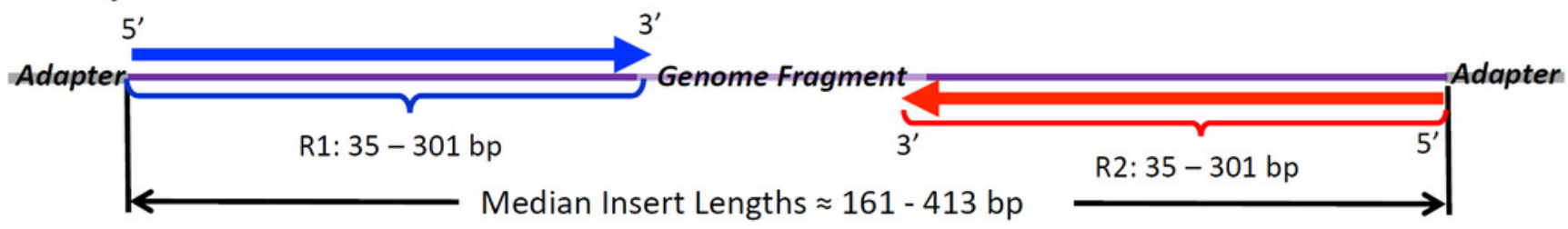

300-cycle

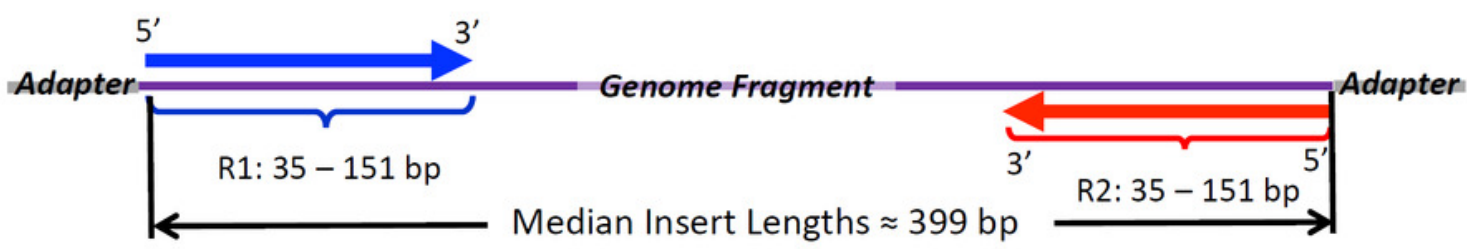


Figure 2

MSA Lengths from Unambiguous SNPs.

(A) SNPs forE. coli O26 (Cluster 1) with Lyve-SET (dark grey bars) and CFSAN_SNP_Pipeline (light gray bars). (B) SNPs for Salmonella enterica Reading (Cluster 2) with Lyve-SET and CFSAN_SNP_Pipeline. (C) SNPs for S. enterica Pomona (Cluster 3) with Lyve-SET and CFSAN_SNP_Pipeline. (D) SNPs for Shigella sonnei (Cluster 4) with Lyve-SET and CFSAN_SNP_Pipeline. For both Lyve-SET and CFSAN across all four clusters, SNPs were counted from final multiple sequence alignments including positions with ambiguous nucleotides. 

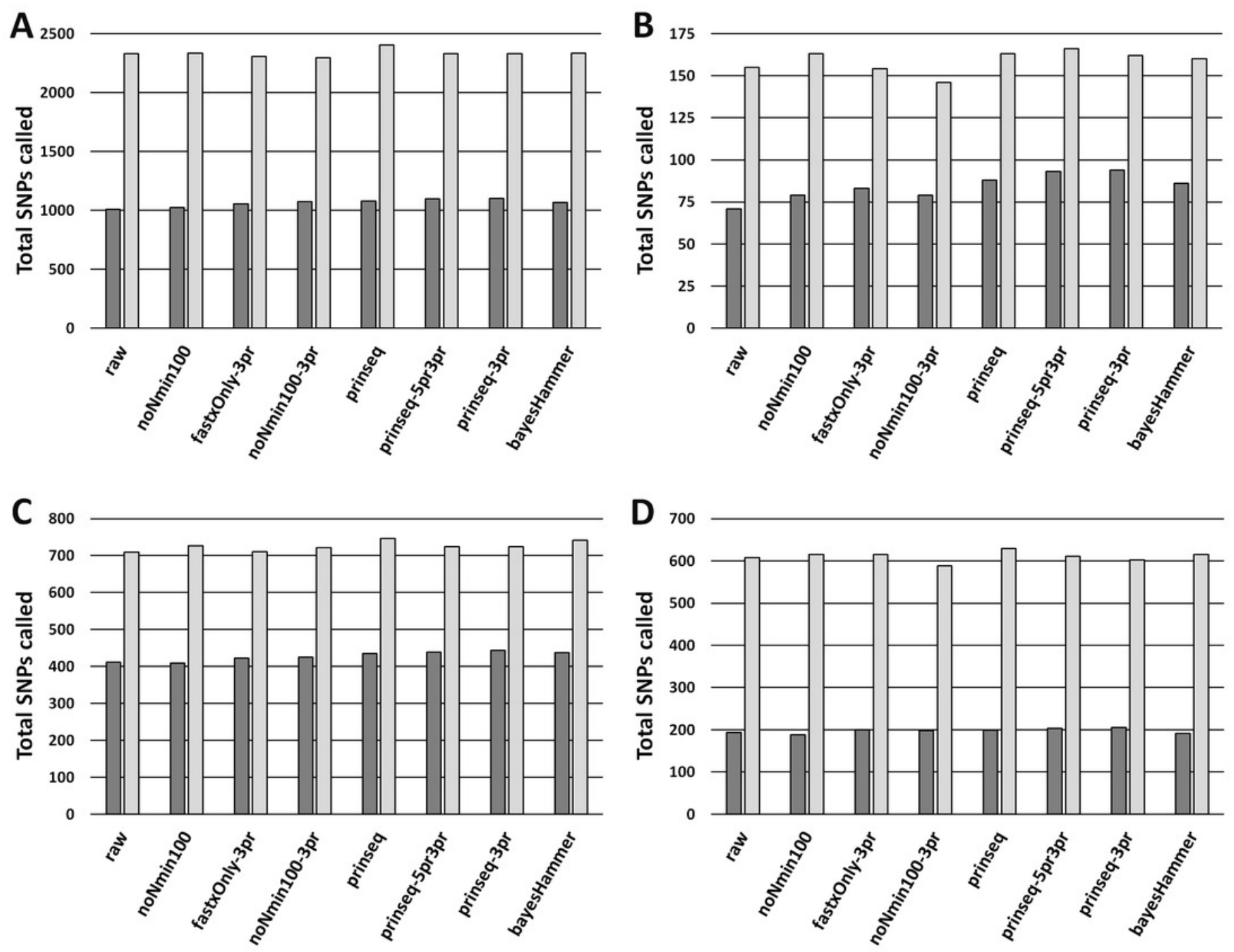
Figure 3

Read Healing Effects on SNPs Identification

ROC-like plots of unique Lyve-SET SNPS (estimated false discovery rate in Data S5) compared to detected concordant SNPs or True Positive Rate (estimated sensitivity in Data S5). (A) E. coli 026 (Cluster 1). (B) S. enterica serovar Reading (Cluster 2). (C) S. enterica serovar Pomona (Cluster 3). (D) S. sonnei (Cluster 4). Estimated false discovery and True Positive Rate for the CFSAN SNP Pipeline are plotted in Figure S5. 
A

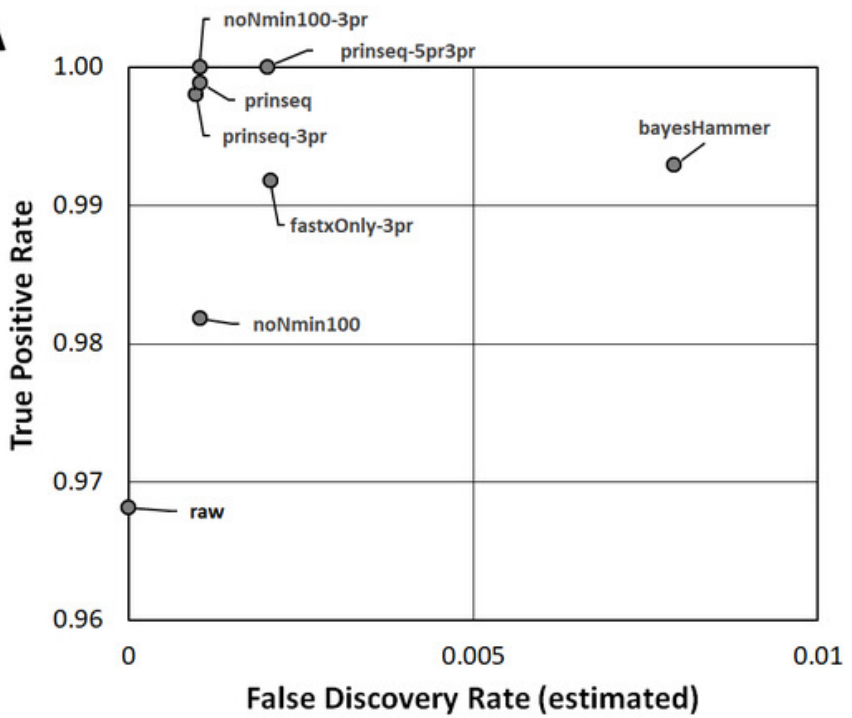

C

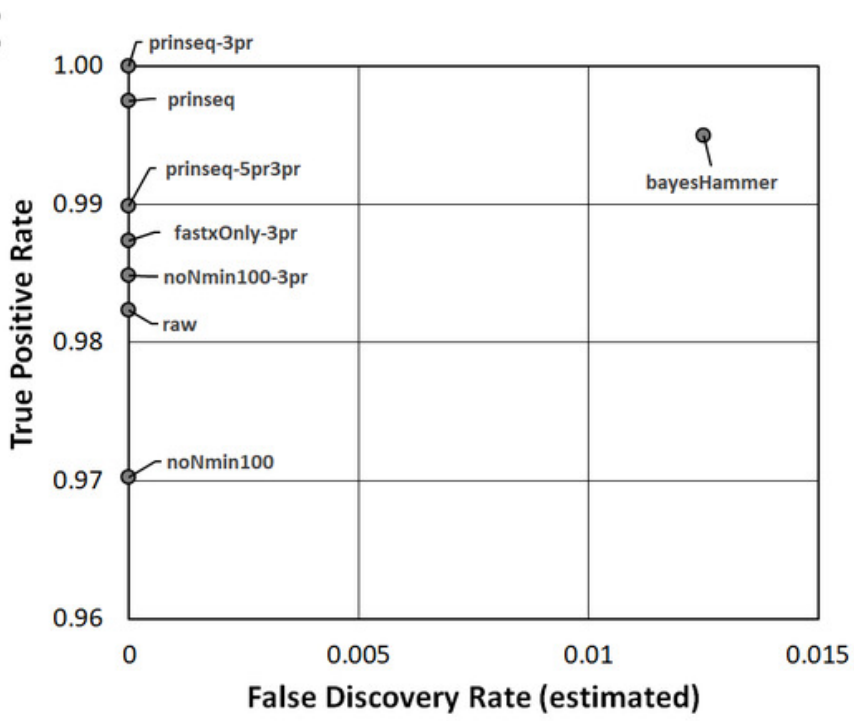

B

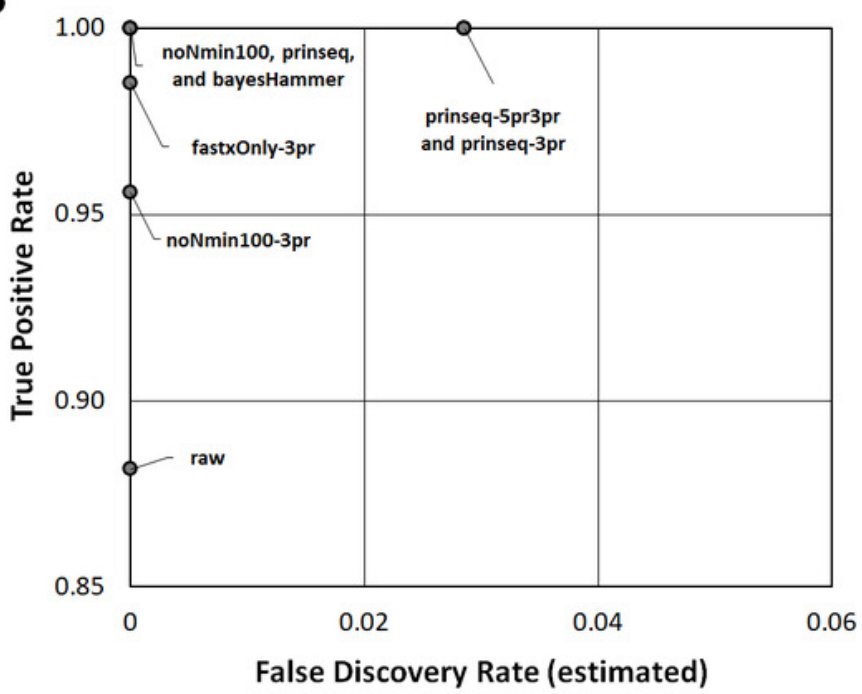

D

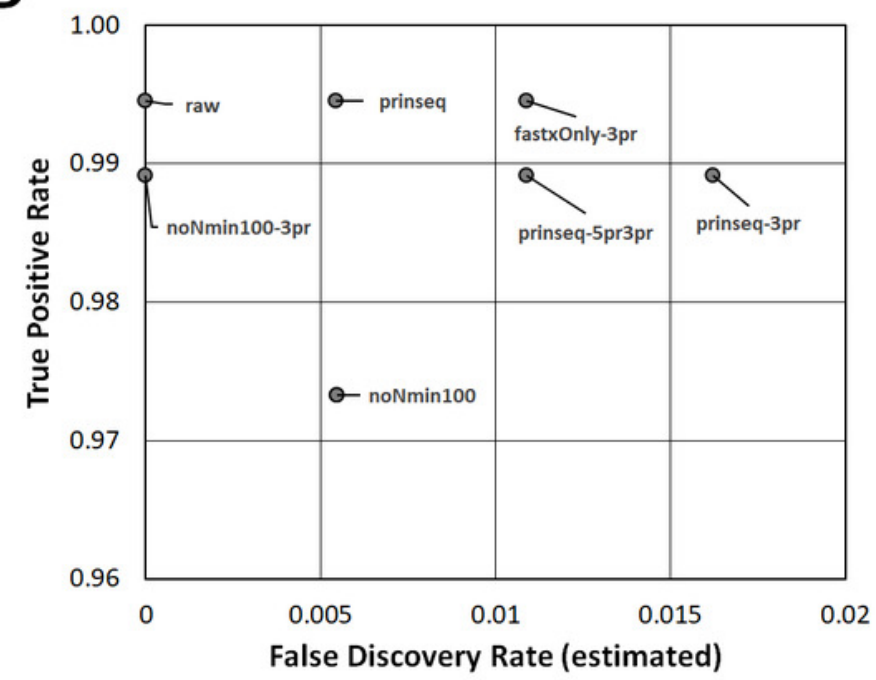




\section{Table $\mathbf{1}$ (on next page)}

Read quality metrics: R1/R2 PHRED quality, median insert lengths, and percentage of $R 1+R 2$ reads with Ns.

Range of averages quality scores for reads from isolates and inserts; medians are shown in parentheses. Results of healing pipelines are shown per data set. noNmin100 not shown due to lack of statistically significant results when compared to raw reads. 
1 Table 1. Read quality metrics: R1/R2 PHRED quality, median insert lengths, and

2 percentage of $\mathbf{R} 1+\mathbf{R} 2$ reads with Ns. Range of averages quality scores for reads from isolates

3 and inserts; medians are shown in parentheses. Results of healing pipelines are shown per data

4 set. noNmin100 not shown due to lack of statistically significant results when compared to raw

5 reads.

6

7

\begin{tabular}{|c|c|c|c|c|c|}
\hline & & $\begin{array}{l}\text { E. coli ser. } \mathbf{O 2 6} \\
\text { (Cluster 1) }\end{array}$ & $\begin{array}{c}\text { S. enterica ser. } \\
\text { Reading (Cluster 2) }\end{array}$ & $\begin{array}{c}\text { S. enterica ser. } \\
\text { Pomona (Cluster 3) }\end{array}$ & $\begin{array}{l}\text { Shigella sonnei } \\
\quad \text { (Cluster 4) }\end{array}$ \\
\hline \multirow{4}{*}{ 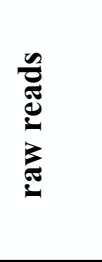 } & R1 qual. & $33.0-36.1(34.8)$ & $32.5-35.7(34.2)$ & $32.2-36.9(35.3)$ & $34.2-36.8(35.9)$ \\
\hline & R2 qual. & $26.0-33.5(30.3)$ & $27.5-32.8(30.2)$ & $26.1-35.3(32.2)$ & $30.2-35.4(33.9)$ \\
\hline & insert (bp) & $161-371(264)$ & $175-531(290)$ & $184-556(383)$ & $212-492(309)$ \\
\hline & $\mathrm{R} 1+\mathrm{R} 2 \mathrm{Ns} \%$ & $0.0-5.1$ & $0.0-4.6$ & $0.0-31.9$ & $0.0-12.2$ \\
\hline \multirow{4}{*}{ 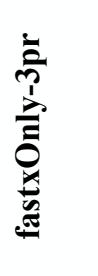 } & R1 qual. & $33.1-36.1(34.8)$ & $32.8-35.3(34.4)$ & $32.3-36.9(35.3)$ & $34.3-36.9(35.9)$ \\
\hline & R2 qual. & $26.5-33.8(30.8)$ & $27.6-32.8(30.4)$ & $26.8-35.6(32.6)$ & $30.5-35.6(34.2)$ \\
\hline & insert (bp) & $171-372(268)$ & $176-532(293)$ & $198-558(389)$ & $213-493(310)$ \\
\hline & $\mathrm{R} 1+\mathrm{R} 2 \mathrm{Ns} \%$ & $0.0-4.7$ & $0.0-4.5$ & $0.0-31.9$ & $0.0-3.2$ \\
\hline \multirow{4}{*}{ 总 } & R1 qual. & $34.4-36.7(35.7)$ & $34.5-36.7(35.4)$ & $33.9-37.2(36.0)$ & $35.0-37.2(36.5)$ \\
\hline & R2 qual. & $30.8-35.0(32.9)$ & $31.4-34.6(33.2)$ & $30.8-36.2(34.2)$ & $33.0-36.2(35.3)$ \\
\hline & insert (bp) & $192-382(283)$ & $187-539(296)$ & $229-564(406)$ & $219-494(312)$ \\
\hline & $\mathrm{R} 1+\mathrm{R} 2 \mathrm{Ns} \%$ & $0.0-3.5$ & $0.0-3.2$ & $0.0-1.5$ & $0.0-3.7$ \\
\hline \multirow{4}{*}{ 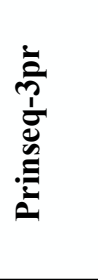 } & R1 qual. & $34.5-36.7(35.8)$ & $34.5-36.7(35.5)$ & $34.1-37.2(36.1)$ & $35.0-37.2(36.5)$ \\
\hline & R2 qual. & $31.1-35.2(33.2)$ & $31.6-34.8(33.4)$ & $31.3-36.3(34.4)$ & $33.1-36.3(35.4)$ \\
\hline & insert (bp) & $192-383(283)$ & $187-546(296)$ & $229-564(406)$ & $220-494(312)$ \\
\hline & $\mathrm{R} 1+\mathrm{R} 2 \mathrm{Ns} \%$ & $0.0-3.4$ & $0.0-3.2$ & $0.0-1.5$ & $0.0-2.3$ \\
\hline \multirow{4}{*}{ 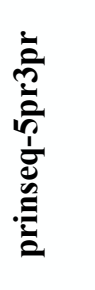 } & R1 qual. & $34.5-36.7(35.8)$ & $34.6-36.8(35.6)$ & $34.1-37.2(36.1)$ & $35.0-37.3(36.5)$ \\
\hline & R2 qual. & $31.1-35.2(33.2)$ & $31.6-35.2(33.6)$ & $31.3-36.3(34.4)$ & $33.1-36.3(35.5)$ \\
\hline & insert (bp) & $190-380(281)$ & $183-538(293)$ & $227-562(404)$ & $218-492(310)$ \\
\hline & $\mathrm{R} 1+\mathrm{R} 2 \mathrm{Ns} \%$ & $0.0-3.4$ & $0.0-3.1$ & $0.0-1.4$ & $0.0-2.3$ \\
\hline \multirow{3}{*}{ 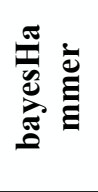 } & R1 qual. & $33.1-36.1(34.9)$ & $32.6-35.7(34.2)$ & $32.3-36.9(35.3)$ & $34.2-36.9(35.9)$ \\
\hline & R2 qual. & $26.8-33.6(30.5)$ & $27.6-32.8(30.3)$ & $26.3-35.4(32.3)$ & $30.4-35.5(34.0)$ \\
\hline & insert (bp) & $189-412(290)$ & $182-540(300)$ & $227-587(401)$ & $216-499(314)$ \\
\hline
\end{tabular}




\begin{tabular}{lllll}
\hline & $\begin{array}{c}\text { E. coli ser. O26 } \\
\text { (Cluster 1) }\end{array}$ & $\begin{array}{c}\text { S. enterica ser. } \\
\text { Reading (Cluster 2) }\end{array}$ & $\begin{array}{c}\text { S. enterica ser. } \\
\text { Pomona (Cluster 3) }\end{array}$ & $\begin{array}{c}\text { Shigella sonnei } \\
\text { (Cluster 4) }\end{array}$ \\
\hline $\mathrm{R} 1+\mathrm{R} 2 \mathrm{Ns} \%$ & $0.0-1.7$ & $0.0-0.6$ & $0.0-32.0$ & $0.0-6.5$ \\
\hline
\end{tabular}

8

9

10 


\section{Table 2 (on next page)}

Assembly quality metrics average values.

Boldface underlined values indicate one-sided Dunn post-hoc test improvement over raw read scores at $\alpha=0.05$ level of significance (with Benjamani-Hochberg correction). Reads healed through pipelines; noNmin100 and fastxOnly-3pr did not produce assemblies with statistically significant improvements over any metric and are not shown. 
1 Table 2. SPAdes Assembly quality metrics average values. Boldface underlined values

2 indicate one-sided Dunn post-hoc test improvement over raw read scores at $\alpha=0.05$ level of

3 significance (with Benjamani-Hochberg correction). Reads healed through pipelines;

4 noNmin100 and fastxOnly-3pr did not produce assemblies with statistically significant

5 improvements over any metric and are not shown.

6

\begin{tabular}{|c|c|c|c|c|c|c|c|}
\hline & $\begin{array}{c}\text { Metric } \\
\text { (Kruskal- } \\
\text { Wallis p) }\end{array}$ & $\begin{array}{c}\text { Raw } \\
\text { Reads }\end{array}$ & $\begin{array}{c}\text { noNmin } 100- \\
3 p r\end{array}$ & prinseq & $\begin{array}{l}\text { prinseq - } \\
5 \text { pr3pr }\end{array}$ & prinseq-3pr & bayesHammer \\
\hline \multirow{3}{*}{$\begin{array}{c}\text { E. coli } \\
\text { O26 } \\
\text { (Cluster 1) } \\
\text { Kruskal- } \\
\text { Wallis } \\
\text { df }=7\end{array}$} & $\begin{array}{c}\text { Contigs } \\
\left(1.95 \times 10^{-10}\right)\end{array}$ & 330.5 & 318.6 & $\left(0.00 \frac{\mathbf{2 9 5 . 9}}{6916)}\right.$ & $(0.006258)$ & $(0.009774)$ & (0.00 $\frac{\mathbf{2 8 7 . 2}}{1382)}$ \\
\hline & $\begin{array}{c}\text { N50 } \\
(0.01364)\end{array}$ & $81,036.8$ & $84,793.3$ & $92,749.2$ & $92,574.8$ & $\begin{array}{r}\mathbf{9 3 , 1 4 0 . 8} \\
(0.04853)\end{array}$ & $92,391.1$ \\
\hline & $\begin{array}{l}\text { Maximum } \\
\text { contig } \\
(0.06474)\end{array}$ & $218,070.1$ & $213,638.3$ & $245,939.0$ & $239,557.8$ & $250,158.6$ & $251,436.1$ \\
\hline \multirow{3}{*}{$\begin{array}{c}\text { S. enterica } \\
\text { ser. } \\
\text { Reading } \\
\text { (Cluster 2) } \\
\text { df }=7\end{array}$} & $\begin{array}{l}\text { Contigs } \\
(0.6353)\end{array}$ & 96.0 & 91.8 & 77.5 & 76.8 & 76.5 & 86.0 \\
\hline & $\begin{array}{c}\text { N50 } \\
(0.9972)\end{array}$ & $243,104.2$ & $265,306.8$ & $270,566.3$ & $277,891.2$ & $270,003.6$ & $253,005.8$ \\
\hline & $\begin{array}{l}\text { Maximum } \\
\text { contig } \\
(0.9552)\end{array}$ & $552,949.7$ & $625,007.5$ & $653,148.7$ & $658,147.9$ & $602,089.9$ & $581,313.7$ \\
\hline \multirow{3}{*}{$\begin{array}{c}\text { S. enterica } \\
\text { ser. } \\
\text { Pomona } \\
\text { (Cluster 3) } \\
\text { df }=7\end{array}$} & $\begin{array}{l}\text { Contigs } \\
(0.506)\end{array}$ & 44.5 & 44.2 & 35.5 & 35.3 & 34.7 & 35.6 \\
\hline & $\begin{array}{c}\text { N50 } \\
(0.9714)\end{array}$ & $412,213.0$ & $431,232.0$ & $412,871.0$ & $431,778.0$ & $465,935.0$ & 431,969 \\
\hline & $\begin{array}{c}\text { Maximum } \\
\text { contig } \\
(0.5936)\end{array}$ & $911,229.0$ & $838,277.0$ & $1,000,451.0$ & $1,019,369.0$ & $1,023,669.0$ & $1,039,598$ \\
\hline \multirow{3}{*}{$\begin{array}{c}\text { Shigella } \\
\text { sonnei } \\
\text { (Cluster 4) } \\
\text { df }=7\end{array}$} & $\frac{\text { Contigs }}{(0.5547)}$ & 449.8 & 448.2 & 445.1 & 448.0 & 446.2 & 443.4 \\
\hline & $\begin{array}{c}\text { N50 } \\
(0.8932)\end{array}$ & $23,931.1$ & $24,054.5$ & $24,117.1$ & $23,931.1$ & $23,929.4$ & $24,009.8$ \\
\hline & $\begin{array}{l}\text { Maximum } \\
\text { contig } \\
(0.999)\end{array}$ & $89,893.8$ & $89,378.5$ & $90,046.7$ & $89,870.1$ & $89,632.6$ & $89,352.8$ \\
\hline
\end{tabular}

